\title{
Chasing discs around O-type (proto)stars: Evidence from ALMA observations
}

\author{
R. Cesaroni ${ }^{1}$, Á. Sánchez-Monge ${ }^{2}$, M. T. Beltrán ${ }^{1}$, K. G. Johnston ${ }^{3}$, L. T. Maud ${ }^{4}$, L. Moscadelli ${ }^{1}$, J. C. Mottram ${ }^{5}$, \\ A. Ahmadi ${ }^{5}$, V. Allen ${ }^{6}$, H. Beuther ${ }^{5}$, T. Csengeri ${ }^{7}$, S. Etoka ${ }^{8}$, G. A. Fuller ${ }^{9}{ }^{10}$, D. Galli ${ }^{1}$, R. Galván-Madrid ${ }^{11}$, \\ C. Goddi ${ }^{12,4}$, T. Henning ${ }^{5}$, M. G. Hoare ${ }^{13}$, P. D. Klaassen ${ }^{14}$, R. Kuiper ${ }^{15}$, M. S. N. Kumar ${ }^{16,17}$, S. Lumsden $^{18}$, \\ T. Peters ${ }^{19}$, V. M. Rivilla ${ }^{1}$, P. Schilke ${ }^{2}$, L. Testi ${ }^{20,1}$, F. van der Tak ${ }^{21,6}$, \\ S. Vig ${ }^{22}$, C. M. Walmsley ${ }^{23,1}$, and H. Zinnecker ${ }^{24}$
}

(Affiliations can be found after the references)

Received 2 December 2016 / Accepted 30 January 2017

\begin{abstract}
Context. Circumstellar discs around massive stars could mediate the accretion onto the star from the infalling envelope, and could minimize the effects of radiation pressure. Despite such a crucial role, only a few convincing candidates have been provided for discs around deeply embedded O-type (proto)stars.

Aims. In order to establish whether disc-mediated accretion is the formation mechanism for the most massive stars, we have searched for circumstellar, rotating discs around a limited sample of six luminous $\left(>10^{5} L_{\odot}\right)$ young stellar objects. These objects were selected on the basis of their IR and radio properties in order to maximize the likelihood of association with disc+jet systems.

Methods. We used ALMA with $\sim 0^{\prime \prime 2} 2$ resolution to observe a large number of molecular lines typical of hot molecular cores. In this paper we limit our analysis to two disc tracers (methyl cyanide, $\mathrm{CH}_{3} \mathrm{CN}$, and its isotopologue, ${ }^{13} \mathrm{CH}_{3} \mathrm{CN}$ ), and an outflow tracer (silicon monoxide, $\mathrm{SiO}$ ).

Results. We reveal many cores, although their number depends dramatically on the target. We focus on the cores that present prominent molecular line emission. In six of these a velocity gradient is seen across the core, three of which show evidence of Keplerian-like rotation. The SiO data reveal clear but poorly collimated bipolar outflow signatures towards two objects only. This can be explained if real jets are rare (perhaps short-lived) in very massive objects and/or if stellar multiplicity significantly affects the outflow structure. For all cores with velocity gradients, the velocity field is analysed through position-velocity plots to establish whether the gas is undergoing rotation with $v_{\text {rot }} \propto R^{-\alpha}$, as expected for Keplerian-like discs.

Conclusions. Our results suggest that in three objects we are observing rotation in circumstellar discs, with three more tentative cases, and one core where no evidence for rotation is found. In all cases but one, we find that the gas mass is less than the mass of any embedded O-type star, consistent with the (putative) discs undergoing Keplerian-like rotation. With the caveat of low number statistics, we conclude that the disc detection rate could be sensitive to the evolutionary stage of the young stellar object. In young, deeply embedded sources, the evidence for discs could be weak because of confusion with the surrounding envelope, while in the most evolved sources the molecular component of the disc could have already been dispersed. Only in those objects that are at an intermediate stage of the evolution would the molecular disc be sufficiently prominent and relatively less embedded to be detectable by $\mathrm{mm} / \mathrm{submm}$ observations.
\end{abstract}

Key words. stars: early-type - stars: formation - ISM: molecules

\section{Introduction}

How do massive stars form? A growing number of observational and theoretical studies are focused on this topic. Recent years have seen significant progress (Tan et al. 2014) and we can now claim that numerical simulations adequately describe the formation of the most massive stars (Krumholz et al. 2007a; Kuiper et al. 2010; Peters et al. 2010). Observations are still lagging behind, but the dramatic improvement in sensitivity and/or resolution offered by a number of facilities in recent years (Herschel, NOEMA, ALMA) is now allowing a quantum leap in our knowledge of the formation process of all stars in general, and of earlytype stars in particular.

As in the low-mass case, discs around OB-type stars play an important role in angular momentum transfer (Rosen et al. 2012), but their existence is particularly crucial in allowing accretion to proceed, overcoming the powerful radiation pressure of luminous protostars (Krumholz et al. 2009; Kuiper et al. 2011; Peters et al. 2011; Klassen et al. 2016). In their comprehensive review, Beltrán \& de Wit (2016) have summarized the current evidence for discs around intermediate-mass ( 2$\left.7 M_{\odot}\right)$ and high-mass $\left(\gtrsim 7 M_{\odot}\right)$ stars, from the embedded to the optically visible phase. Comparison between this recent review and the previous one by Cesaroni et al. (2007) shows that while little doubt can now be cast on the existence of discs around B-type (proto)stars, those around O-type stars remain elusive. Observational evidence for massive cores, possibly undergoing (solid-body) rotation and containing newly born early-type stars has been provided by various authors (see e.g. Beltrán \& de Wit 2016; Zapata et al. 2015). However, despite the prediction that Keplerian rotation could be seen at up to $1000 \mathrm{AU}$ radii (see Fig. 4 of Kuiper et al. 2011), only one convincing case is known of a Keplerian disc around a deeply embedded (proto)star exceeding the approximate threshold of $\sim 15 M_{\odot}$ separating B- from O-type stars (Mottram et al. 2011b). This is AFGL 4176, for which Johnston et al. (2015) derive a stellar mass of $25 M_{\odot}$ corresponding to an 07 spectral type. Not surprisingly, this result has been obtained thanks to the resolution and sensitivity of the Atacama Large Millimeter/submillimeter Array (ALMA). 
In fact, prior to the finding of Johnston and collaborators, ALMA had already been crucial in placing the existence of discs around B-type stars on solid ground. While evidence for such discs was provided by various authors (see Cesaroni et al. 2007; Beltrán \& de Wit 2016, and references therein), it was unclear whether disc-mediated accretion is the standard mechanism for the formation of B-type stars. With this in mind, we performed ALMA observations of two selected targets, with luminosities of $\sim 10^{4} L_{\odot}$ and associated with bipolar nebulae and hot molecular core (HMC) tracers. Our observations confirmed the presence of Keplerian discs, rotating about stellar masses of 9-18 $M_{\odot}$ (Sánchez-Monge et al. 2013, 2014; Beltrán et al. 2014), which indicates a continuity in the formation process from solar-type to B-type stars.

Based on this successful project, we have taken the study a step further and extended our disc search to the most massive stars. All models predict the existence of discs around such stars, although it is possible that their structure, size, and lifetime depend upon the formation process. Circumstellar discs are a natural outcome of the Bondi accretion process depicted by Keto $(2002,2003,2007)$ and maintain their overall structure and velocity field even while partly ionized. The monolithicaccretion theory by McKee \& Tan (2003) - in its modern incarnations by Krumholz et al. (2007b, 2009) - implies highly fragmented discs, encompassing binary and even multiple systems. More critical could be the situation of discs in the competitive accretion scenario by Bonnell et al. (2006), where a highly dynamical process favours the formation of the most massive stars in the gravitational well of a rich stellar cluster. Such a perturbed scenario could have even more dramatic consequences for the disc structure than just fragmentation: discs might be truncated or destroyed and reformed on relatively short (dynamical) timescales. In conclusion, the observable disc parameters can potentially provide insights into the OB-type star formation process itself.

With all of the above in mind, we performed ALMA observations of a selected sample of luminous $\left(>10^{5} L_{\odot}\right)$ young stellar object (YSOs) to investigate the presence of discs and possibly establish their characteristics. This article summarizes the preliminary findings of our study, while a detailed description of each source will be given in forthcoming dedicated papers. In the following, we explain how the targets were selected (Sect. 2), describe the observations and data reduction (Sect. 3), present the main results (Sect. 4), and analyse our findings to draw conclusions on the existence of discs around O-type (proto)stars (Sects. 5 and 6). Finally, a summary is given in Sect. 7.

\section{Target selection and characterization}

Our goal is to establish whether disc accretion is the common mechanism for the formation of O-type stars. We thus chose six targets from the "toroid" sample of Beltrán et al. (2005, 2011a) and the RMS survey database ${ }^{1}$ (Lumsden et al. 2013; Urquhart et al. 2008), which satisfy most of the following criteria:

- luminosity $>10^{5} L_{\odot}$, the minimum value for O-type stars;

- presence of a jet/outflow, from previous studies and/or data available to us, based on the assumption that discs are associated with bipolar flows ejected along the disc rotation axis;

- known association with a hot molecular core and/or mid-IR colours typical of young massive (proto)stars $\left(S_{21 \mu \mathrm{m}} / S_{8 \mu \mathrm{m}} \gtrsim 2\right.$; Lumsden et al. 2013);

\footnotetext{
1 http://rms.leeds.ac.uk/cgi-bin/public/RMS_DATABASE. cgi
}

- distance below $8 \mathrm{kpc}$, to maximize the number of feasible targets without reducing the spatial resolution too much.

The six targets are given in Table 1 with their equatorial coordinates, local standard of rest (LSR) velocity $\left(V_{\mathrm{LSR}}\right)$, bolometric luminosity $\left(L_{\mathrm{bol}}\right)$, mass of the molecular clump ${ }^{2}$ enshrouding the corresponding star forming region $\left(M_{\mathrm{gas}}\right)$, and distance $(d)$. The values of $L_{\text {bol }}$ for G17.64, G345.49, and G345.50 have been taken from the RMS database ${ }^{3}$. For G29.96 we used the estimate of Beltrán et al. (2013), based on the Hi-GAL data, scaled to the new distance estimate of $5.26 \mathrm{kpc}$ (Zhang et al. 2014). For G24.78 and G31.41, we estimated $L_{\text {bol }}$ by integrating under the spectral energy distribution reconstructed using the data from various surveys (Hi-GAL/Herschel, WISE, MSX, MIPSGAL/Spitzer). The value of $M_{\text {gas }}$ was computed from the flux density at $870 \mu \mathrm{m}$ obtained from the ATLASGAL ${ }^{4}$ data (Schuller et al. 2009; Contreras et al. 2013), assuming an arbitrary dust temperature $T_{\text {dust }}=50 \mathrm{~K}$, a dust absorption coefficient $\kappa_{\text {dust }}=2 \mathrm{~cm}^{2} \mathrm{~g}^{-1}$ at $870 \mu \mathrm{m}$ (Ossenkopf \& Henning 1994), and a gas-to-dust mass ratio of 100 .

Three sources lie at $\sim 2 \mathrm{kpc}$ and the other three at $\sim 6-8 \mathrm{kpc}$. While this makes it difficult to compare the properties of the different objects, it allows us to investigate possible biases related to the distance.

We can get a hint of the evolutionary stage of the star forming region to which the targets belong by inspecting the far-IR colour temperature, $T_{\mathrm{c}}[160-70]$, between 70 and $160 \mu \mathrm{m}$ and the ratio $L_{\mathrm{bol}} / M_{\mathrm{gas}}$. The latter is believed to be a good proxy for the source age (see e.g. McKee \& Tan 2003; Elia et al. 2010), as the gas mass is converted into stars during the process of star formation. The former is very sensitive to the temperature of the clump enshrouding the star forming region, as the typical spectral energy distribution of high-mass YSOs peaks around $\sim 100 \mu \mathrm{m}$ (see e.g. van der Tak 2000; Cesaroni et al. 2015). Since the environment is progressively heated by the newly formed stars, an increase in the dust temperature with time and thus an increase in $T_{\mathrm{c}}[160-70]$ with age is expected. In Fig. 1, we plot these two parameters for our targets, for which we obtained the farIR fluxes from the Hi-GAL data (Molinari et al. 2010, 2016). Indeed both parameters give consistent age indications: colder sources have lower $L_{\mathrm{bol}} / M_{\text {gas }}$ ratios, and vice versa. On this basis, the targets can be grouped into three pairs with progressively increasing age: G24.78 and G31.41; G345.49 and G345.50; and G17.64 and G29.96.

A word of caution is in order for the luminosity and mass estimates. The former assume isotropic emission, but inclination with respect to the line of sight could play a role if the clumps are not spherically symmetric and opacity is non-negligible. For example, the flashlight effect can lead to underestimating the luminosity by an order of magnitude (see Zhang et al. 2013) if the bipolar structure is seen edge-on. Conversely, a pole-on view may cause a significant overestimate of the luminosity. Our estimated luminosity may also be affected by the radiation from nearby more evolved massive stars, which are only loosely connected with the target of interest. An illuminating example is

\footnotetext{
2 We refer to "clumps" as pc-scale structures, as opposed to "cores", whose sizes are below $\sim 0.1 \mathrm{pc}$.

3 The RMS luminosities were derived using the method described in Mottram et al. (2011a), updated with the Hi-GAL/Herschel far-IR fluxes (Molinari et al. 2010, 2016) where available (Mottram et al., in prep.).

4 The ATLASGAL project is a collaboration between the Max-PlanckGesellschaft, the European Southern Observatory (ESO), and the Universidad de Chile.
} 
R. Cesaroni et al.: Chasing discs around O-type (proto)stars: Evidence from ALMA observations

Table 1. Targets for the ALMA observations with their main parameters, obtained from the literature.

\begin{tabular}{|c|c|c|c|c|c|c|c|c|}
\hline Name & $\begin{array}{l}\text { Other } \\
\text { names }\end{array}$ & $\begin{array}{c}\alpha(\mathrm{J} 2000) \\
(\mathrm{h} \mathrm{m} \mathrm{s})\end{array}$ & $\begin{array}{c}\delta(\mathrm{J} 2000) \\
\left({ }^{\circ}, \prime \prime\right)\end{array}$ & $\begin{array}{c}V_{\mathrm{LSR}} \\
\left(\mathrm{km} \mathrm{s}^{-1}\right)\end{array}$ & $\begin{array}{c}d \\
(\mathrm{kpc})\end{array}$ & $\begin{array}{c}L_{\mathrm{bol}} \\
\left(10^{5} L_{\odot}\right)\end{array}$ & $\begin{array}{c}M_{\operatorname{gas}^{a}} \\
\left(10^{3} M_{\odot}\right)\end{array}$ & $\operatorname{Ref}^{c}$ \\
\hline G17.64+0.16 & AFGL 2136 & 182226.370 & -133012.00 & 22.5 & 2.2 & 1.0 & 0.20 & 1 \\
\hline $\mathrm{G} 24.78+0.08$ & & 183612.661 & -071210.15 & 111.0 & 7.7 & 2.2 & 4.8 & 2,3 \\
\hline G29.96-0.02 & W43 S & 184603.665 & -023922.00 & 98.0 & 5.26 & 5.8 & 1.3 & 4,5 \\
\hline $\mathrm{G} 31.41+0.31$ & & 184734.315 & -011245.90 & 96.5 & 7.9 & 2.6 & 5.2 & 3,6 \\
\hline G345.49+1.47 & & 165941.610 & -400343.30 & -12.6 & $2.4^{b}$ & 1.5 & 1.2 & 1 \\
\hline G345.50+0.35 & & 170422.870 & -404423.50 & -17.8 & 2.0 & 1.0 & 0.55 & 1 \\
\hline
\end{tabular}

Notes. ${ }^{(a)}$ Computed from the flux density at $870 \mu \mathrm{m}$ obtained from the ATLASGAL data, assuming a dust temperature of $50 \mathrm{~K}$, a dust absorption coefficient $\kappa_{\text {dust }}=2 \mathrm{~cm}^{2} \mathrm{~g}^{-1}$ (Ossenkopf \& Henning 1994), and a gas-to-dust mass ratio of 100 . ${ }^{(b)}$ We prefer the spectrophotometric distance estimate by Moisés et al. (2011) to the kinematic distance of $1.6 \pm 0.1 \mathrm{kpc}$ by Urquhart et al. (2008). (c) References for distance and luminosity: 1. RMS database; 2. Forster \& Caswell (1989); 3. this paper; 4. Beltrán et al. (2013); 5. Zhang et al. (2014); 6. Cesaroni et al. (1994a).

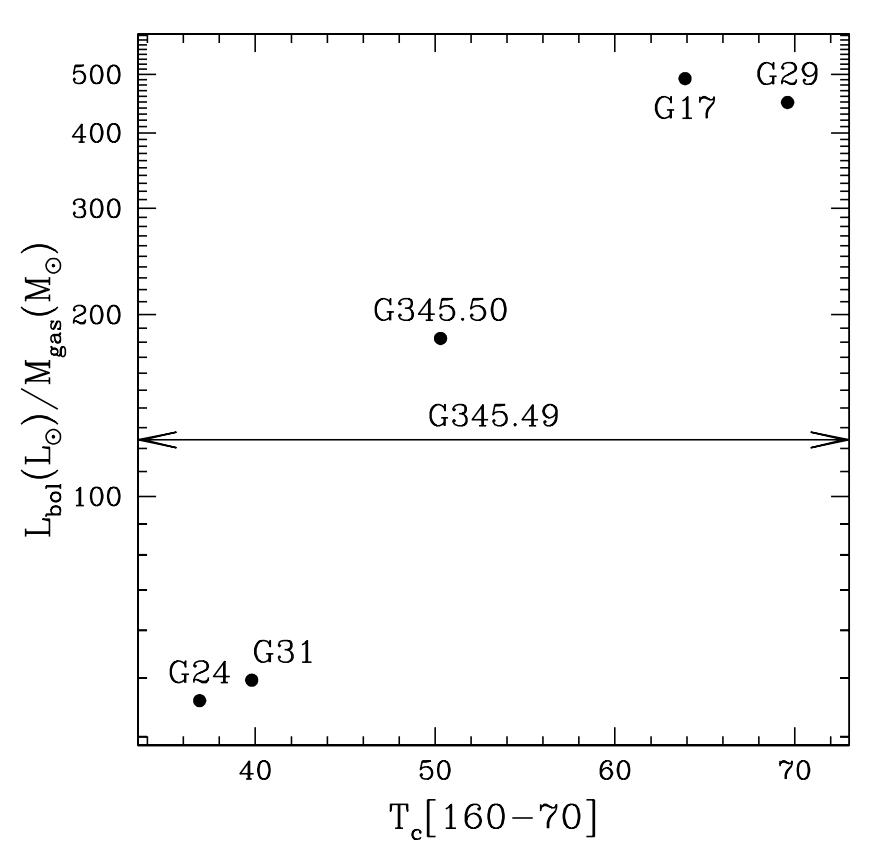

Fig. 1. Plot of the ratio between the bolometric luminosity and the mass of the associated molecular clump vs. the colour temperature obtained from the ratio between the far-IR flux densities at 70 and $160 \mu \mathrm{m}$, for all the sources of our sample. The horizontal line denotes that for source G345.49 no estimate of $T_{\mathrm{c}}[160-70]$ is possible because the source lies beyond the region covered by the Hi-GAL survey.

that of G29.96. Although de Buizer (2002) has demonstrated that the HMC is a prominent mid-IR emitter, the nearby ultracompact HII region is known to be associated with an O5-O6 star (Martín-Hernández et al. 2003), which may therefore contribute to a significant fraction of $L_{\text {bol }}$ (up to $92 \%$; see Vacca et al. 1996). The value of $5.8 \times 10^{5} L_{\odot}$ is thus an upper limit to the luminosity of the HMC. While a similar conclusion may hold also for the other five cores, the contribution from nearby stars is rather uncertain in these cases, as such stars have not been directly observed.

Finally, we note that a priori the mass estimate can be affected by significant errors (a factor of $\sim 2$; see Maud et al. 2015a), mostly due to the uncertainties on $\kappa_{\text {dust }}$ and $T_{\text {dust }}$. However, the latter is unlikely to vary more than $T_{\mathrm{c}}[160-70]$, namely $\sim 40 \%$, while the former should not change much from source to source because the selected sample is relatively homogeneous. Assuming that $\kappa_{\text {dust }}$ is indeed the same in all sources,
Table 2. Spectral windows covered by the correlator set-up, with corresponding spectral resolutions and noise per channel.

\begin{tabular}{|c|c|c|c|}
\hline $\begin{array}{c}\text { Frequency range } \\
(\mathrm{GHz})\end{array}$ & $\begin{array}{c}\text { Resolution } \\
(\mathrm{kHz})\end{array}$ & $\begin{array}{c}1 \sigma \text { noise }^{a} \\
(\mathrm{mJy} / \text { beam })\end{array}$ & Molecule $^{b}$ \\
\hline $216.976-218.849$ & 1953.1 & 1.3 & $\mathrm{SiO}$ \\
\hline 219.533-219.767 & 488.3 & 1.5 & \\
\hline $220.303-220.537$ & 244.1 & 2.0 & $\mathrm{CH}_{3} \mathrm{CN}$ \\
\hline $220.533-220.767$ & 244.1 & 1.9 & $\mathrm{CH}_{3} \mathrm{CN}$ \\
\hline $221.213-221.447$ & 488.3 & 1.8 & $\mathrm{CH}_{3} \mathrm{CN} v_{8}=1$ \\
\hline $231.803-232.037$ & 488.3 & 1.8 & ${ }^{13} \mathrm{CH}_{3} \mathrm{CN}$ \\
\hline 232.033-232.267 & 488.3 & 1.6 & ${ }^{13} \mathrm{CH}_{3} \mathrm{CN}$ \\
\hline $232.903-233.137$ & 488.2 & 1.7 & \\
\hline $233.133-233.367$ & 488.3 & 1.5 & \\
\hline $235.283-235.517$ & 488.3 & 1.4 & \\
\hline $235.853-236.087$ & 488.3 & 1.6 & \\
\hline 236.083-236.317 & 488.3 & 1.5 & \\
\hline 236.313-236.547 & 488.3 & 1.6 & \\
\hline
\end{tabular}

Notes. ${ }^{(a)}$ The noise has been estimated in the channel corresponding to the strongest emission in the bandwidth. This value is only indicative, as it can change significantly from source to source. ${ }^{(b)}$ Only the species analysed in the present study are reported.

the points in Fig. 1 can only shift systematically along the $y$-axis, thus preserving the observed trend.

\section{Observations and data analysis}

ALMA was employed to perform observations towards the positions given in Table 1 . The number of $12 \mathrm{~m}$ antennas used ranged from 38 to 41 . The correlator was set in such a way to cover a large number of lines, with special attention to the $\mathrm{CH}_{3} \mathrm{CN}(12-$ $11),{ }^{13} \mathrm{CH}_{3} \mathrm{CN}(13-12)$, and $\mathrm{SiO}(5-4)$ rotational transitions. A broad $1.8 \mathrm{GHz}$ spectral unit was also used to obtain a sensitive continuum measurement at $\sim 218 \mathrm{GHz}$. The primary beam of the $12 \mathrm{~m}$ antennas at this frequency is $\sim 26^{\prime \prime}$. The frequency range of each spectral unit and the corresponding spectral resolution and sensitivity are given in Table 2, where we also indicate which spectral units cover the species discussed in the present article. We note that the frequency coverage is not exactly the same for all sources, because pairs of targets were observed in track sharing mode, which requires a compromise for the LSR velocity. 
The observations were conducted during Cycle 2 in July and September 2015, with the array in an extended configuration with baselines ranging from $40 \mathrm{~m}$ to $1500 \mathrm{~m}$. Flux calibration was obtained through observations of the planetary system bodies Titan and Ceres. The phases were calibrated from interleaved observations of the quasars J1733-1304 (for G17.64 and G24.78), J1851+0035 (for G29.96 and G31.41), and J17093525 (for G345.49 and G345.50). The gain calibrators, separated from the sources on the sky by $2^{\circ}-5^{\circ}$, were observed every $6 \mathrm{~min}$. The bandpass correction was obtained by observing the bright quasars J1733-1304, J1617-5848 (in July), and J19242914 (in July and September). The on-source observing time per source was about $50 \mathrm{~min}$.

The calibration and imaging were carried out in CASA. Imaging was performed on each spectral window using the CLEAN task, with the robust parameter of Briggs (1995) set to 0.5, as a compromise between resolution and sensitivity to extended emission. The resulting images have a synthesized CLEANed beam of about $0^{\prime \prime} .2$. The rms noise level in a spectral channel is given in Table 2, while in the continuum maps the noise ranges from 0.2 to $1 \mathrm{mJy} /$ beam and is mostly limited by the dynamic range.

\subsection{Continuum image and continuum subtraction}

The usual method used to create an image of the continuum emission and to subtract it from the data consists in identifying the channels where no line emission is present, under the assumption that these channels correspond to the continuum level. Subtraction can then be performed in the uv or image plane. While this method can be easily adopted for sources with a relatively low level of line emission, in our case almost all the observed spectra present a "line forest", which makes the visual identification of a continuum level extremely difficult.

We thus decided to employ a statistical method which estimates the continuum level at each position of the map from the distribution of the intensity in the different channels of the spectrum at that position. This is done within the python-based tool STATCONT (Sánchez-Monge et al. 2017), which is freely accessible $^{5}$. In practice, we compute the median, $\mu$, and standard deviation, $\sigma$, of this distribution, and assume that the continuum level is equal to $\mu-\sigma+\sigma_{0}$, where $\sigma_{0}$ is the mean value of the noise over a region void of emission. This correction is needed because the expression $\mu-\sigma$ would result in a negative value in those positions where no emission is detected, and in all cases it would underestimate the correct continuum emission. In this process, the strongest channels are progressively excluded through an iterative procedure (known as sigma clipping), where all channels whose flux lies beyond the interval $\mu \pm 2 \sigma$ are not used in the next step of the calculation of $\mu$ and $\sigma$. The loop stops when the values of $\mu$ and $\sigma$ no longer change. The continuum image obtained in this way is then subtracted from the corresponding data cube.

The validity of our procedure is described in detail and tested with numerical simulations in Sánchez-Monge et al. (2017). We note that the method is applied to each spectral window of the correlator, independently of the others. This makes it acceptable to neglect the dependence of the continuum emission on frequency since the bandwidths are relatively narrow $(<2 \mathrm{GHz})$.

http://www . astro.uni-koeln.de/ sanchez/statcont

\section{Results}

In this section, we present and discuss the main findings of our observations, focusing on the comparison between the different sources. A more detailed analysis of each target will be performed in a number of dedicated papers.

\subsection{Continuum emission}

The maps of the continuum emission of the six targets are shown in Fig. 2. All of these maps were obtained from the spectral unit with the largest bandwidth $(1.8 \mathrm{GHz})$, using the procedure described in Sect. 3.1. Most of the maps present a clumpy structure; the emission is concentrated in a single source in only two cases (G17.64 and G31.41). In particular, in G17.64 the continuum source is basically unresolved with a synthesized beam of $\sim 0^{\prime \prime} .15$, and the emission could entirely be due to free-free emission, based on the results of Menten \& van der Tak (2004). The arc-like feature in G29.96 traces the rim of the cometary ultracompact (UC) HII region and is (at least partly) due to freefree continuum emission from the ionized gas. Prominent freefree emission is also contaminating one source in G24.78 (see Beltrán et al. 2007) and one in G345.49 (Guzmán et al. 2010), known from previous observations at centimetre wavelengths. All three of these continuum sources (G29.96, G24.78, G345.49) are also clearly detected in the $\mathrm{H} 30 \alpha$ recombination line, which is covered by our frequency set-up.

All the other features detected above a $5 \sigma$ level in Fig. 2 are dominated by dust emission and trace compact molecular cores. It thus appears that the level of fragmentation in the observed regions varies quite a lot, from compact objects such as G17.64 and G31.41 (although on different spatial scales) to very clumpy regions like G24.78 and G345.49, where visual inspection identifies up to nine fragments in each of them. In G31.41, a large continuum opacity could justify the (apparent) homogeneity of the core, but even so, the putative fragments would be "squeezed" inside a more compact region than in the other targets. The variety of clumpiness is better illustrated in Fig. 3, where the same data as in Fig. 2 are shown with constant physical scales. The most distant fields such as G31.41 might indeed contain more undetected cores, but the different morphologies observed in the various sources cannot be explained by the different spatial resolutions alone. This is further emphasized in Fig. 4, where the fraction of the continuum flux density recovered by the interferometer is plotted as a function of the source distance. For this purpose we have divided the integrated flux density over the whole ALMA map by the flux density measured inside a $33^{\prime \prime}$ beam (similar to the ALMA primary beam) towards the phase centre in the corresponding BOLOCAM GPS image (Ginsburg et al. 2013). For the two sources not covered in the BOLOCAM survey (G345.49 and G345.50) we note that we used the flux density measured by Faúndez et al. (2004) at $1.2 \mathrm{~mm}$. For consistency with the BOLOCAM measurements, the fluxes were rescaled to a HPBW of 33" by assuming a Gaussian source with the full width at half maximum measured by Faúndez and collaborators.

While on average the fraction of recovered flux in the ALMA data increases with distance to the source, a large spread is present at all distances. For example, both G24.78 and G31.41 are located at $\sim 8 \mathrm{kpc}$, but an order of magnitude less flux is recovered for the former region than for the latter. This indicates that variations in the level of clumpiness are real and not entirely due to different distances and mass sensitivities. 


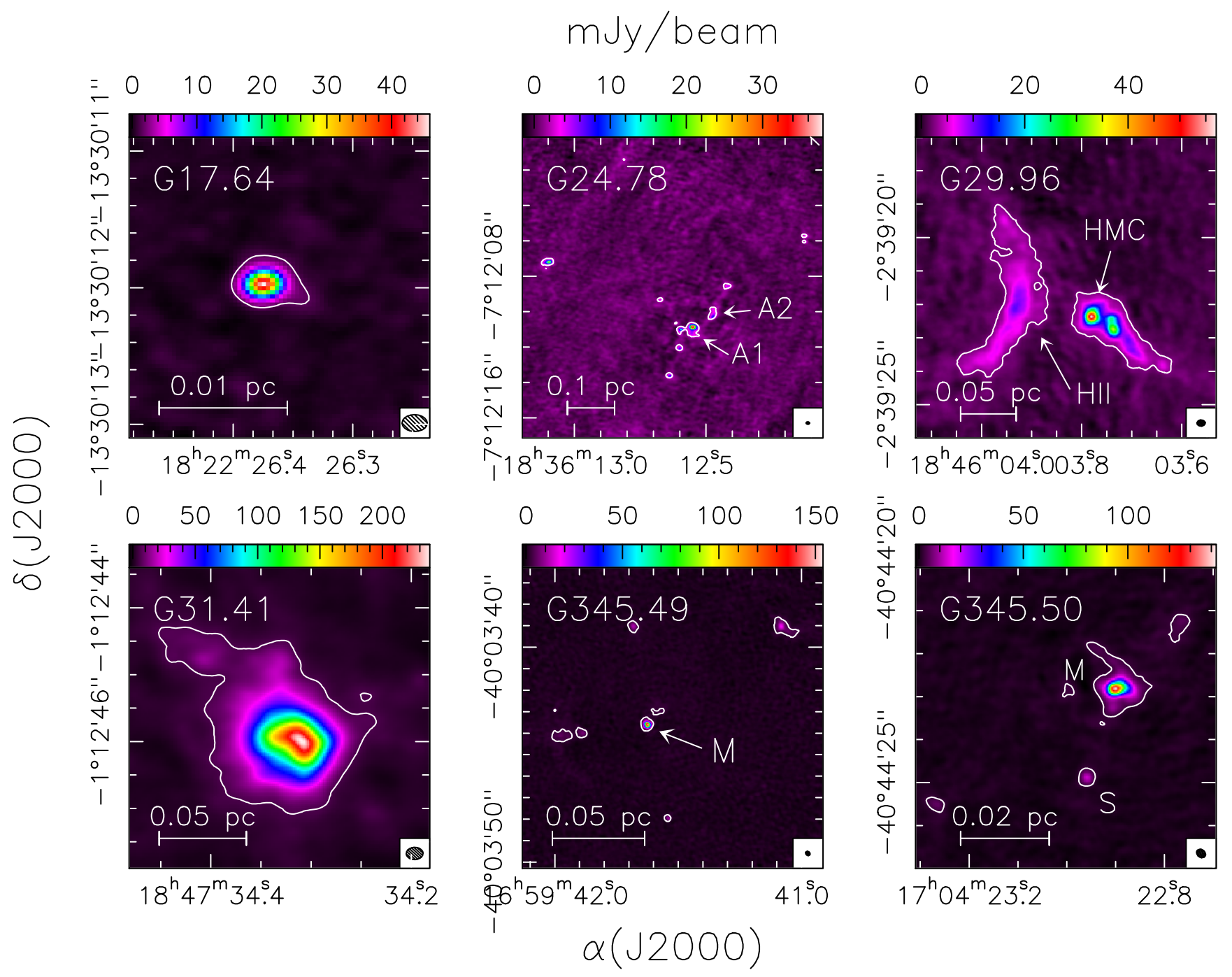

Fig. 2. Maps of the continuum emission at $218 \mathrm{GHz}$ from the six targets observed. The solid contours correspond to the $5 \sigma$ levels. The ellipse in the bottom right corner of each panel denotes the half-power width of the synthesized beam. The maps cover the whole region where emission is detected. The labels indicate the UC HII region in G29.96 and the cores that we will refer to in the present study. The maximum recoverable scale in these images is $\sim 4^{\prime \prime}$, much greater than the diameter of the largest cores detected.

In conclusion, despite the similar luminosities, our targets are quite heterogeneous both in terms of evolutionary stage (see Sect. 2) and level of fragmentation.

\subsection{Line emission}

In Fig. 5 we show a template spectrum obtained by integrating the line emission from the HMC in G29.96, inside the contour corresponding to the $5 \sigma$ level of the continuum map in Fig. 2. This spectrum clearly illustrates the plethora of lines covered by our set-up.

The line identification process is a difficult and timeconsuming task that goes beyond the scope of the present paper and will be discussed in forthcoming articles. Here, we make only a qualitative comparison among the different targets in terms of line richness and intensity. For this purpose, we define two parameters: $f$, the fraction of spectral channels where line emission is detected, and $I$, the mean intensity per unit bandwidth of the emission in these channels, scaled by $d^{2}$ to take into account the different distances to the sources. We compute $f$ and $I$ for the largest bandwidth $(\sim 1.8 \mathrm{GHz})$ covered in our correlator set-up. In practice, we consider the spectrum towards the peak of the continuum emission. When multiple continuum sources are detected, we select the strongest of them.

Table 3 gives the values obtained for our six targets. The obvious conclusion is that most of the cores are line rich, with the exception of G345.49; G17.64 also seems line poor, but the low value of $f$ is partly due to the fact that it is emission weak. Although its mean intensity is half that of G345.49, the value of $f$ is larger. Moreover, $f$ in G17.64 is only 1.5 times lower than in G24.78, whose $I$ is 19 times stronger. All this suggests that G17.64 is intrinsically more chemically rich than it appears. We come back to this point in Sect. 5.1.

In the following, we concentrate on a few molecular species: $\mathrm{CH}_{3} \mathrm{CN}$ and its isotopologues, and $\mathrm{SiO}$. This choice is dictated by the goal of our study, namely to search for circumstellar discs and the associated bipolar jets/outflows. It is well known that methyl cyanide is an excellent hot core tracer and has proved successful in tracing the dense, hot gas in circumstellar discs around B-type (proto)stars (see e.g. Cesaroni et al. 2014; Sánchez-Monge et al. 2013). Since O-type stars are likely to be enshrouded by even larger and more massive envelopes than B-type stars, we also analyse the isotopologue ${ }^{13} \mathrm{CH}_{3} \mathrm{CN}$, whose transitions are optically thinner and thus better suited 


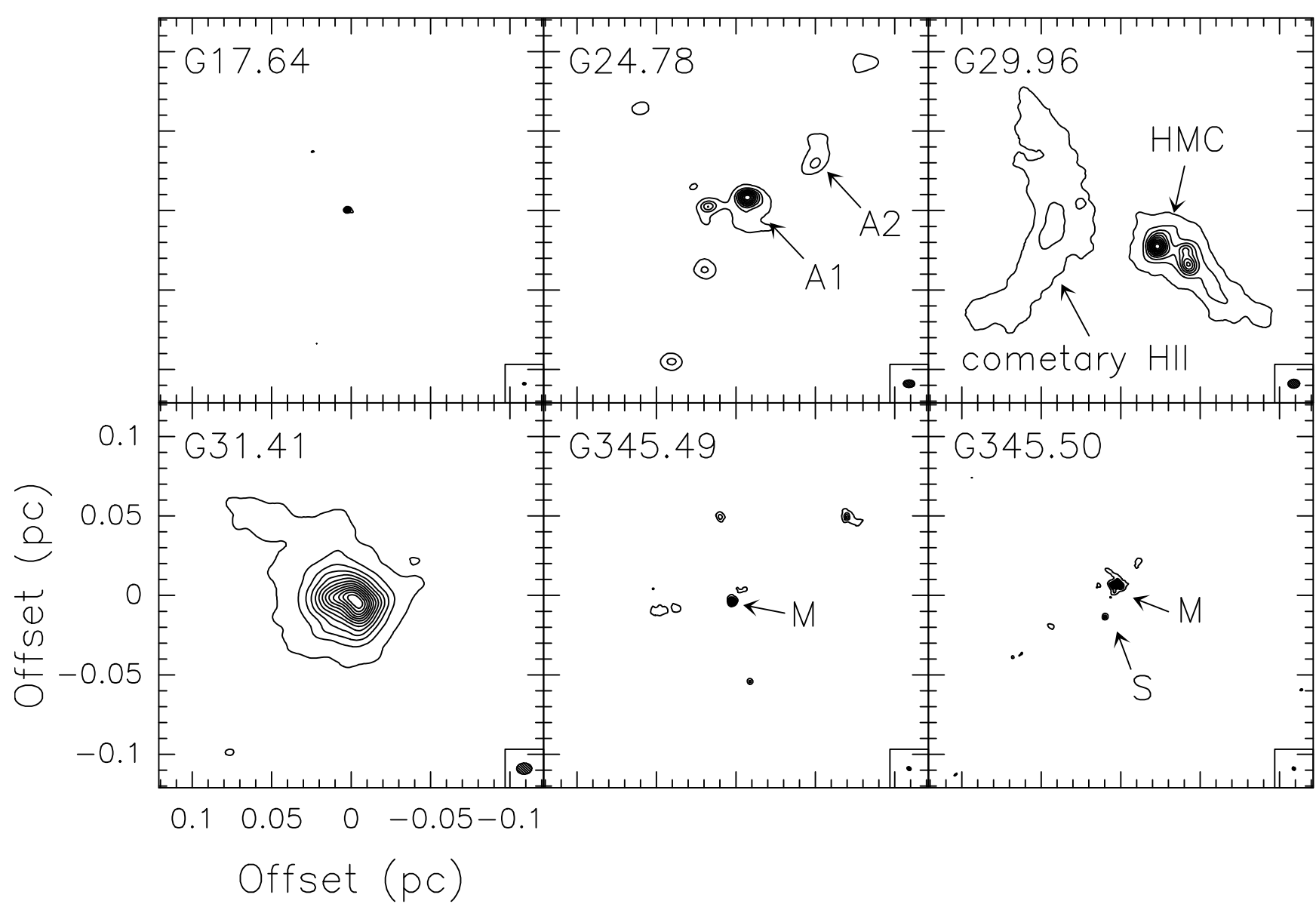

Fig. 3. Same as Fig. 2, with axes expressed in linear scale instead of angular scale. In order to facilitate the visual comparison between the different fields, the size of the region shown is the same for all maps. Symbols have the same meaning as in Fig. 2. The minimum, maximum, and step of the contour levels are 1.5, 43.5, $3 \mathrm{mJy} /$ beam for G17.64; 2.5, 52.5, $5 \mathrm{mJy} /$ beam for G24.78; 2.5, 52.5, $5 \mathrm{mJy} / \mathrm{beam}$ for G29.96; 6, 222, $18 \mathrm{mJy} / \mathrm{beam}$ for G31.41; 2.5, 152.5, $5 \mathrm{mJy} /$ beam for G345.49; and 2.2, 138.6, $4.4 \mathrm{mJy} /$ beam for G345.50. The area shown in the maps lies inside the primary beam, whose half-power width is in all cases $>0.25 \mathrm{pc}$.

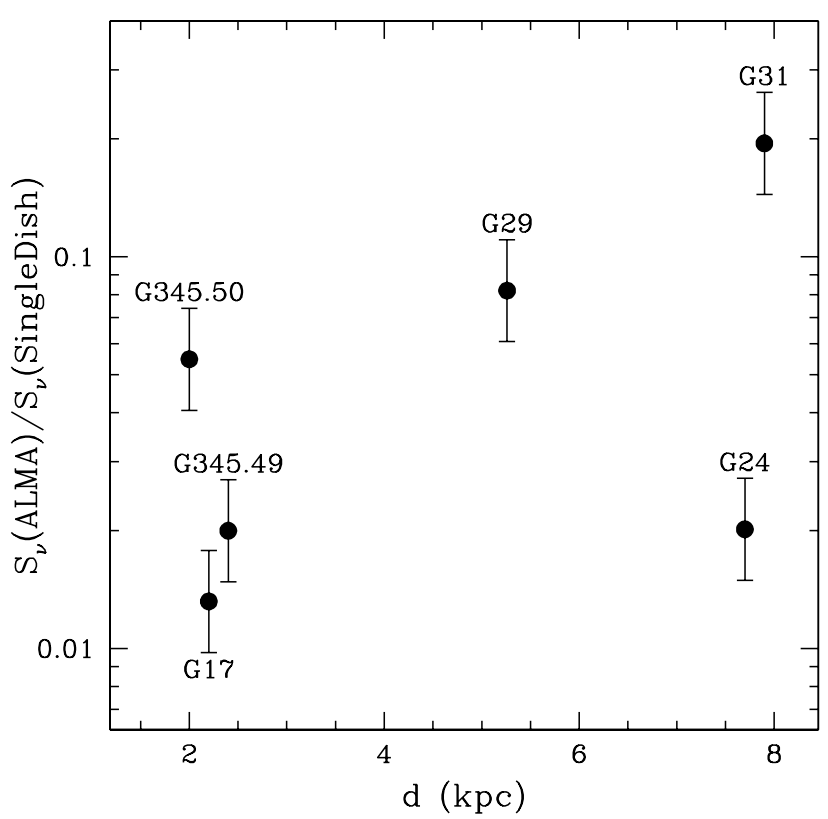

Fig. 4. Ratio between the total continuum flux density recovered by ALMA and that measured in a 33" beam towards the phase centre of each target (obtained from the BOLOCAM GPS images at $1.1 \mathrm{~mm}$ ) vs. source distance. The error bars correspond to $30 \%$ calibration uncertainty on the flux ratio.
Table 3. Richness $(f)$ and mean intensity $(I)$ of the line emission in the observed objects.

\begin{tabular}{lcc}
\hline \hline Name & $\begin{array}{c}f \\
(\%)\end{array}$ & $\begin{array}{c}I \\
\left(\mathrm{mJy} \mathrm{beam}^{-1} \mathrm{kpc}^{2}\right)\end{array}$ \\
\hline G17.64+0.16 & 23 & 0.12 \\
G24.78+0.08 & 35 & 2.31 \\
G29.96-0.02 & 79 & 1.32 \\
G31.41+0.31 & 74 & 2.26 \\
G345.49+1.47 & 17 & 0.25 \\
G345.50+0.35 & 77 & 0.18 \\
\hline
\end{tabular}

to investigating the densest gas close to the star(s). As for the jets/outflows, the interaction between the outflowing gas and the surrounding environment is expected to cause shocks (see e.g. Kuiper et al. 2016), triggering the formation of silicon monoxide, which is indeed often observed in knots along the jet/outflow axis (see e.g. Bachiller et al. 1997; Gueth et al. 1998; Sollins et al. 2004; Cesaroni et al. 2005).

In order to get an idea at first glance of the morphology and kinematics of the dense gas in the circumstellar environment of our sources, in the top panels of Figs. 6-11 we show the map of the average emission over the $K=2$ component of $\mathrm{CH}_{3} \mathrm{CN}(12-11)$ at $220730.266 \mathrm{MHz}$ and the corresponding first and second moment maps for each target. The same maps for 
G29.96-0.02

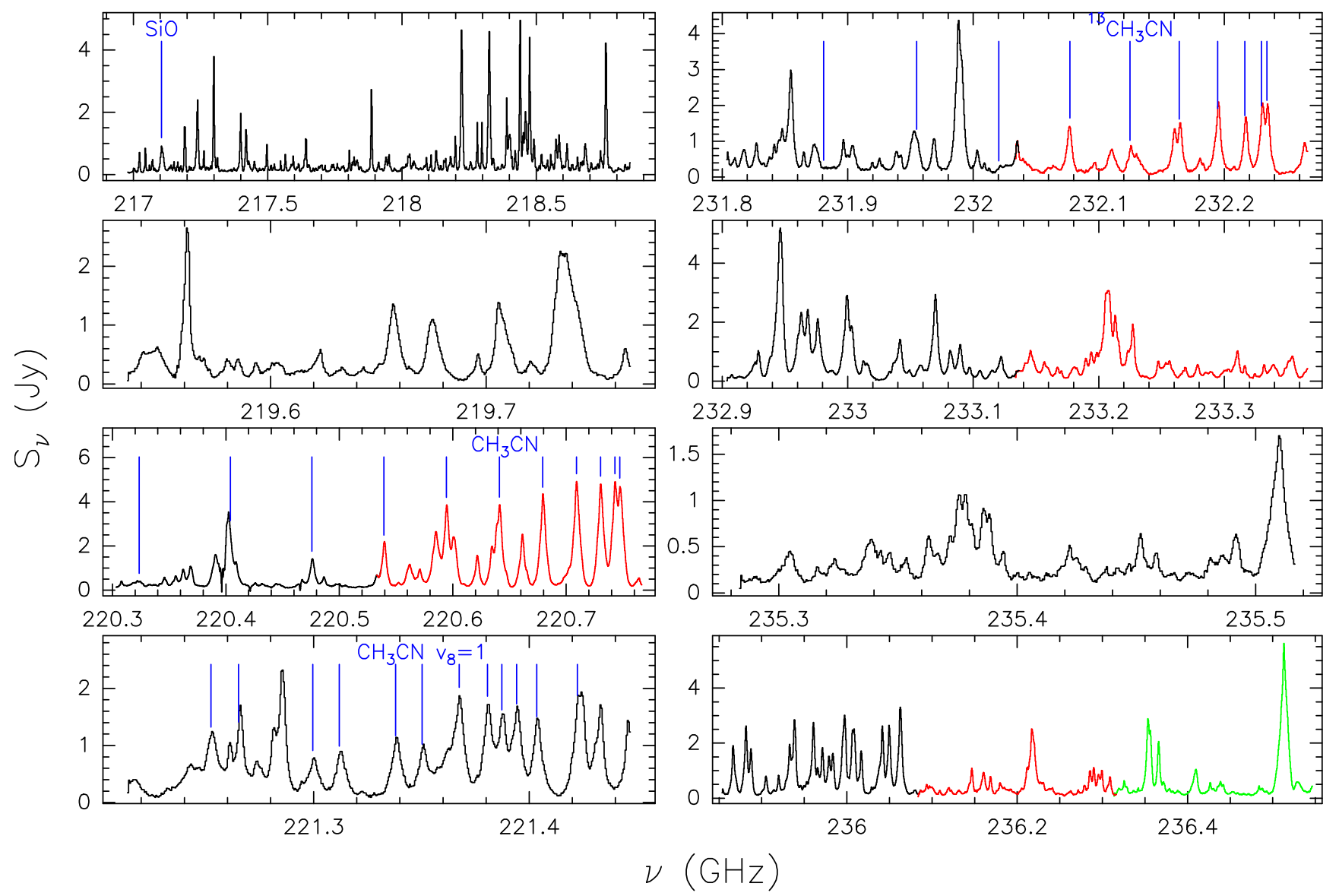

Fig. 5. Spectra obtained by integrating the emission over the $5 \sigma$ contour level of the continuum map of the G29.96 HMC. The whole frequency range covered by our correlator set-up is shown. Different colours in the same box correspond to different spectral windows of the correlator. The vertical lines mark the positions of the $\mathrm{SiO}(5-4), \mathrm{CH}_{3} \mathrm{CN}(12-11)$ ground state, $\mathrm{CH}_{3} \mathrm{CN}(12-11) v_{8}=1$, and ${ }^{13} \mathrm{CH}_{3} \mathrm{CN}(13-12)$ lines. Not all of these are detected and some of them are heavily blended with other transitions.

the ${ }^{13} \mathrm{CH}_{3} \mathrm{CN}(13-12) \mathrm{K}=2$ line at $232216.438 \mathrm{MHz}$ are shown in the bottom panels of the same figures for all sources except G17.64, where no ${ }^{13} \mathrm{CH}_{3} \mathrm{CN}$ transition was detected. For the sake of comparison, we also plot two contours, corresponding to the $5 \sigma$ level and the full width at half maximum (FWHM) of the $1.4 \mathrm{~mm}$ continuum emission. We have chosen the $K=2$ line because it provides us with the highest $\mathrm{S} / \mathrm{N}$ and minimum contamination by other lines. The $K=0$ and 1 components would be better in terms of $\mathrm{S} / \mathrm{N}$, but they heavily overlap each other, which makes them unusable for kinematical studies.

The structure of the methyl cyanide emission varies significantly from source to source, but in all cases, except perhaps G17.64, clear velocity shifts of several $\mathrm{km} \mathrm{s}^{-1}$ are detected in at least one of the cores traced by the continuum emission. These shifts are seen also in the ${ }^{13} \mathrm{CH}_{3} \mathrm{CN}$ maps. While the interpretation of these gradients is not trivial and will be discussed in Sect. 5, here we note that their mere existence indicates that the densest gas is not undergoing randomly oriented motions, but has a well-organized velocity field.

In a disc+jet system the $\mathrm{SiO}$ line should be emitted along the jet axis, namely perpendicular to the $\mathrm{CH}_{3} \mathrm{CN}$ velocity gradient, which is tracing the disc. It is hence interesting to compare the maps of the two species. Unfortunately, the SiO emission could be properly imaged in only four sources (G17.64, G29.96, G31.41, and G345.50). In the other two targets (G24.78 and G345.49) the emission is resolved out by the interferometer, which is not sensitive to structures above a few arcsec. However, previous SMA observations of the $\mathrm{SiO}(5-4)$ line towards G24.78 (Codella et al. 2013), with better sensitivity to extended emission, have revealed a clear bipolar outflow, whose axis is indicated by the dashed line in the middle panel of Fig. 7. Furthermore, in G345.49, Guzmán et al. (2011) have mapped two large-scale bipolar outflows, one of which is associated with a radio jet centred on the main core that we detected, G345.49 M (see Sect. 5.5 and Fig. 2).

As for the four sources detected by us, in G17.64 the SiO emission looks compact and coincides with the continuum core (see Fig. 12), whereas a clear bipolar symmetry is present in G29.96, G31.41, and G345.50 (see Figs. 13-15), although the orientation with respect to the $\mathrm{CH}_{3} \mathrm{CN}$ velocity gradient varies from source to source. Only in G29.96 are the two perpendicular, whereas in $\mathrm{G} 31.41$ the $\mathrm{SiO}$ axis is inclined by $\sim 30^{\circ}$ with respect to the prominent $\mathrm{CH}_{3} \mathrm{CN}$ velocity gradient, and in $\mathrm{G} 345.50$ the situation appears much more complicated.

We note that the $\mathrm{SiO}$ emission does not appear to trace jetlike morphologies. On the contrary, cases like those of G29.96 suggest wide-angle winds. One may speculate that these are caused by interactions with companions inducing, for example, precession of the outflow. This could be taken as indirect evidence of the presence of multiple sources inside the cores. Indeed, Peters et al. (2014) have demonstrated that wideangle winds are created naturally when several stars form in the 


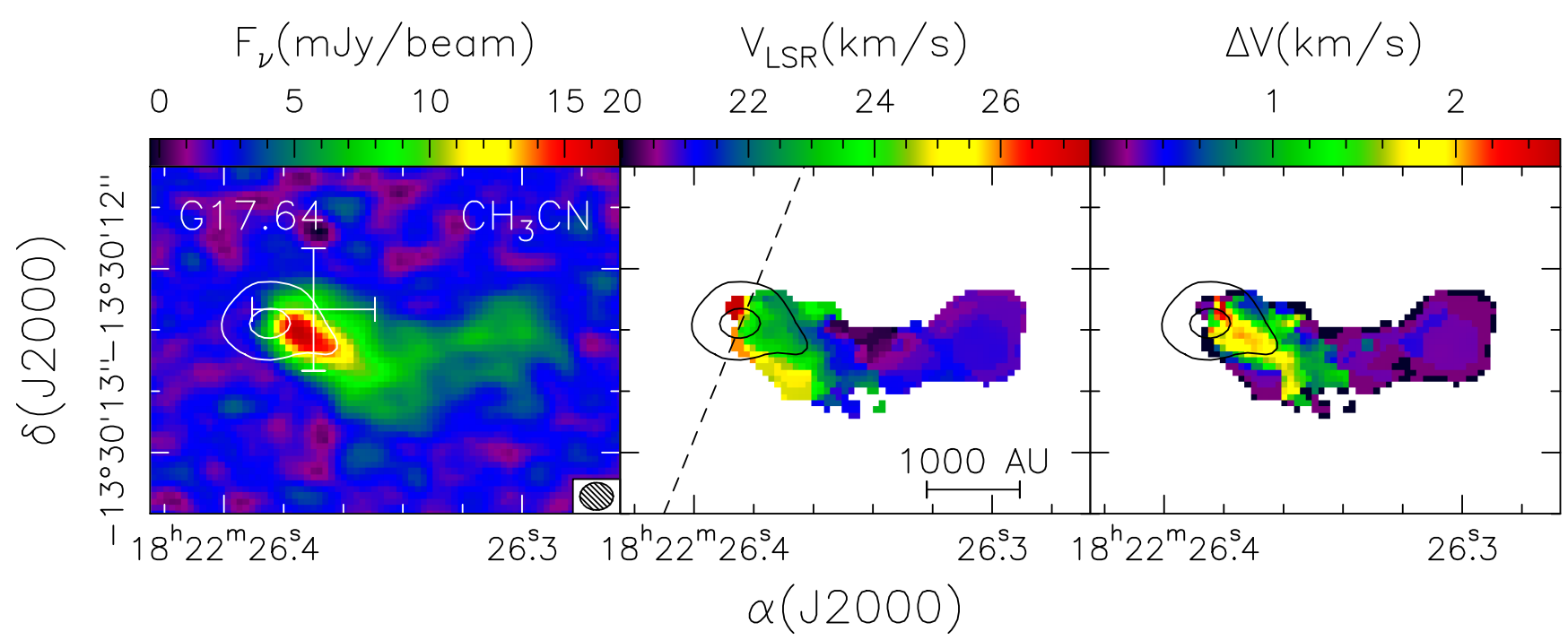

Fig. 6. Left: map of G17.64 obtained by averaging the emission over the $\mathrm{CH}_{3} \mathrm{CN}(12-11) K=2$ line. The contours correspond to the $5 \sigma$ (outer contour) and $50 \%$ level (inner contour) of the continuum emission. The cross marks the position, with errors, of the centimetre continuum source detected by Menten \& van der Tak (2004). The synthesized beam is shown in the bottom right. Middle: same as left panel, but for the first moment map. The dashed line indicates the direction of the axis of the ${ }^{12} \mathrm{CO}$ outflow detected by Kastner et al. (1994). Right: same as left panel, but for the second moment map.

same gravitationally unstable accretion flow. Alternatively, these sources may not currently be driving jets (which could be rare and/or short-lived in these massive YSOs), and the $\mathrm{SiO}$ may be tracing shocks between the wind and the surrounding outflow cavity wall.

\section{Analysis: searching for discs}

Based on the results illustrated above, and despite our attempt to select a homogeneous sample of O-type YSOs, it can be concluded that the observed sources present significant differences even among objects located at similar distances. Such heterogeneity mirrors the complexity of high-mass star forming regions, whose characteristics must depend not only on the properties of the large-scale environment, but also on the evolutionary phase. The structure and even the mere existence of circumstellar discs may be very sensitive to these environmental factors. In the following, we study the dusty cores detected in our targets and investigate the possibility that some of them contain discs around massive stars.

In order to identify a rotating circumstellar disc from the data available to us, two simple criteria can be used: (i) the velocity gradient observed in $\mathrm{CH}_{3} \mathrm{CN}$ should be roughly perpendicular to the associated $\mathrm{SiO}$ jet/outflow axis and (ii) the velocity should increase with decreasing distance to the star. The latter is based on the fact that the gas in accretion discs must fall onto the star conserving the angular momentum or settling in a Keplerian disc. While angular momentum transfer and the presence of turbulence can affect this scenario, it seems reasonable to expect rotation to be faster towards the centre, namely that rotation velocity scales with the distance $R$ from the star as $v_{\text {rot }} \propto R^{-\alpha}$, with $\alpha>0$ ( $\alpha=1 / 2$ corresponds to Keplerian rotation). In the following, we refer to this as "Keplerian-like rotation".

Of course the scenario may be more complicated depending on the gas mass distribution inside $R$, the level of turbulence, the development of instabilities in the disc, and the effect of the magnetic field which could brake the rotation. Therefore, in extreme cases there could be no Keplerian-like motion even at very small radii, where the gravitational field is dominated by the star (see Peters et al. 2011). Despite all these caveats, should we find evidence for $v_{\text {rot }} \propto R^{-\alpha}$, this would hint at the presence of Keplerian-like rotation and thus of a circumstellar disc. This type of rotation curve is recognizable in a position-velocity (PV) plot because the emission outlines a "butterfly-shaped" pattern (see e.g. Cesaroni et al. 2005, 2014).

With this in mind, we analyse the most prominent velocity gradients observed in our sources. The purpose of Sects. 5.1-5.6 is to briefly outline the main characteristics of each source with special attention to the velocity field of the gas, which is the best tool for unveiling the presence of rotation around the embedded star(s). Such a source-by-source description is necessary because of the significant differences among the targets. The results obtained from this analysis will be then summarized and put into context in Sect. 6.

\subsection{G17.64}

In G17.64, also known as AFGL 2136, the continuum source presents only marginal emission of hot-core tracers such as $\mathrm{CH}_{3} \mathrm{CN}$ and the $\mathrm{SiO}$ emission does not appear to be tracing a bipolar flow, as noted in Sect. 4.2. The elongated and bent shape of the $\mathrm{CH}_{3} \mathrm{CN}$ map (see Fig. 6) rather suggests that this molecule might be partially tracing the border of the red-shifted lobe of the bipolar outflow, oriented approximately SE-NW, imaged by Kastner et al. (1994) and Maud et al. (2015b). However, this interpretation is inconsistent with the velocity of most $\mathrm{CH}_{3} \mathrm{CN}$ emission, which appears blue-shifted, whereas the outflow lobe to the NW is red-shifted. An alternative possibility - purely speculative at present - is that the $\mathrm{CH}_{3} \mathrm{CN}$ emission might be tracing infall towards the continuum source. In this scenario, the gas would flow from west to east in the plane of the sky moving towards the observer, thus explaining the blue-shifted emission in Fig. 6. Once the gas has reached the continuum source, it would spiral down onto it making a $180^{\circ}$ turn, which could justify the red-shifted emission at the continuum peak.

In any case, we conclude that no evidence for a circumstellar disc can be obtained from the molecular line emission in this object. Since the source appears to be the most evolved of our 
R. Cesaroni et al.: Chasing discs around O-type (proto)stars: Evidence from ALMA observations

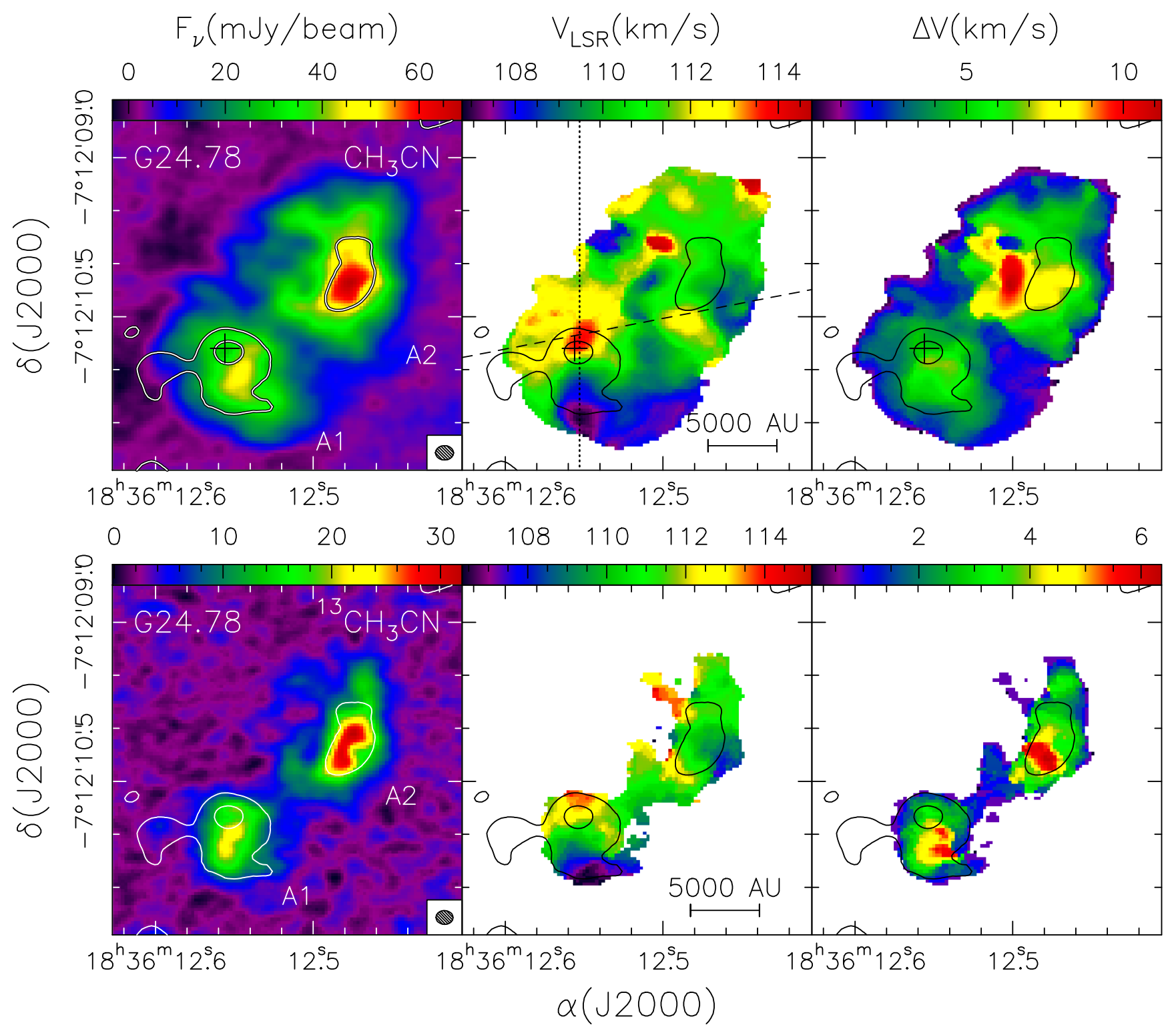

Fig. 7. Top: same as Fig. 6, but for G24.78. The cross in the top panels marks the position of the hypercompact HII region studied by Beltrán et al. (2007) and the dashed line denotes the axis of the bipolar outflow detected by Codella et al. (2013) in the $\mathrm{SiO}(5-4)$ line. The dotted line indicates the direction adopted for the corresponding position-velocity plots. Labels A1 and A2 mark the corresponding HMCs, as in Fig. 2. Bottom: same as top panels, but for the ${ }^{13} \mathrm{CH}_{3} \mathrm{CN}(13-12) K=2$ line.

sample (see Fig. 1), it is possible to speculate that the molecular component of the disc has been substantially dispersed. This hypothesis seems supported by the result discussed in Sect. 4.2, namely that G17.64 is emission weak but chemically rich. Such a chemical complexity is consistent with the (former) presence of a HMC, while the faint line emission indicates that only a remnant of the pristine dense molecular gas is left.

If G17.64 is the most evolved of our sources, one expects to find an HII region associated with it. No radio continuum has been detected at $6 \mathrm{~cm}$ with the VLA at $\sim 2^{\prime \prime}$ resolution (Urquhart et al. 2009; see also the online RMS database), which would hint at a young source. However, Menten \& van der Tak (2004) have revealed a bright $(>2000 \mathrm{~K}$ at $43 \mathrm{GHz}$ ) free-free continuum source, whose position is consistent, within the uncertainties, with the continuum peak seen with ALMA (see Fig. 6). Furthermore, inspection of the corresponding NVSS (Condon et al. 1998) field reveals a peak of continuum emission at $20 \mathrm{~cm}$ of $\sim 3 \mathrm{mJy} / \mathrm{beam}$. These findings suggest that G17.64 could be related to an HII region, although considering the large NVSS image resolution $\left(45^{\prime \prime}\right)$, an association remains ambiguous.

\section{2. $G 24.78$}

Various studies (Beltrán et al. 2004, 2005, 2011b) have provided evidence of up to three rotating cores in the region observed with ALMA. Here, we focus on the most prominent core (A1, following the notation by Beltrán et al. 2005) containing the hypercompact HII region studied by Beltrán et al. (2007) and indicated by a cross in Fig. 7. In this figure it is clear that the gas velocity in the core is red-shifted to the north and blue-shifted to the south, a result which looks different from the NE-SW velocity gradient found by Beltrán et al. (2004, 2005, 2011b). Such a difference is likely due to the different uv coverage of the various data sets. Given the superior image fidelity of ALMA on scales $\lesssim 1^{\prime \prime}$, we believe that our data provide a better representation of the gas structure and motion than those obtained with other (sub)mm 


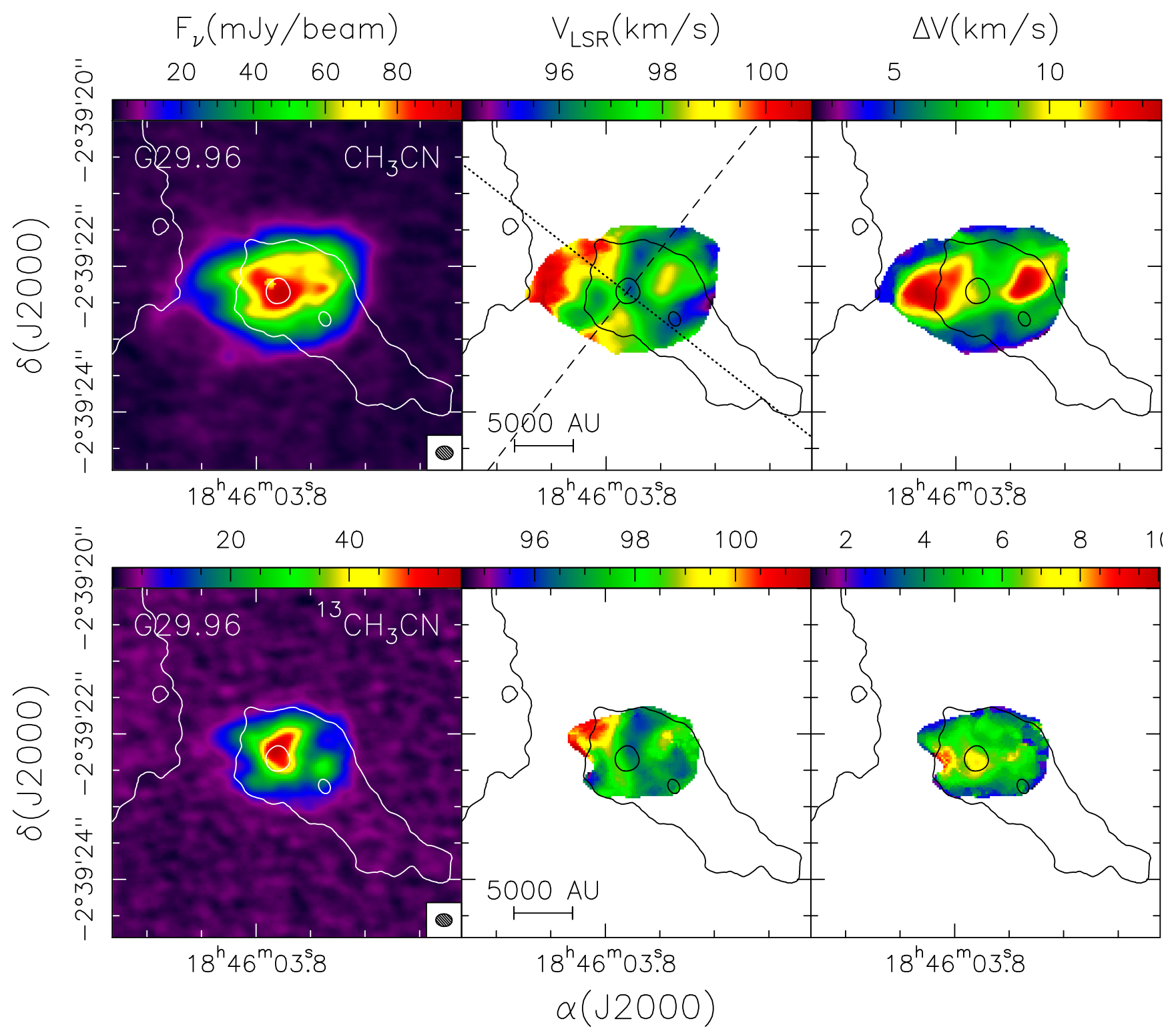

Fig. 8. Same as Fig. 7, but for G29.96. The dashed line marks the direction of the bipolar outflow detected in the present study (see Fig. 13). The dotted line denotes the cut adopted for the PV plot in Fig. 17.

interferometers. We also note that the $\mathrm{N}-\mathrm{S}$ velocity gradient that we detected $\left(\sim 240 \mathrm{~km} \mathrm{~s}^{-1} \mathrm{pc}^{-1}\right)$ appears perpendicular to the $\mathrm{SiO}$ flow imaged by Codella et al. (2013), whose axis is represented by the dashed line in Fig. 7.

While all the above is suggestive of rotation of core A1 about the outflow axis, a couple of caveats are in order.

First of all, according to Codella et al. (2013) and Vig et al. (2008) the outflow is not associated with A1, but the powering source should either coincide with A2 (the other core to the NW where we do not detect a velocity gradient) or lie between A1 and A2. While this is certainly possible, the geometrical centre of a bipolar outflow is difficult to locate with precision and we thus believe that the possibility that A1 is powering the flow cannot be ruled out. Moreover, the existence of a velocity gradient around A1 is important in itself, no matter whether this is associated with the outflow or not.

The second caveat is that the PV plot along the $\mathrm{N}-\mathrm{S}$ direction across the hypercompact HII region (see Fig. 16) looks quite different from the butterfly pattern expected for rotation speed-up toward the star $\left(v_{\text {rot }} \propto R^{-\alpha}\right.$, with $\left.\alpha>0\right)$. For the sake of comparison, in Fig. 16 we also plot the pattern outlining the PV plot of a Keplerian disc (i.e. $\alpha=1 / 2$ ) rotating about a $20 M_{\odot}$ star (the mass of the star ionizing the HII region; Beltrán et al. 2007). There is an excess of emission around $-1^{\prime \prime} .2$ and $106 \mathrm{~km} \mathrm{~s}^{-1}$ (indicated by the arrow in Fig. 16), which seems difficult to reconcile with the Keplerian pattern. However, it is possible that A1 is actually made of two distinct subcores, as suggested by the shape of the continuum emission, which looks elongated $\mathrm{N}-\mathrm{S}$. In this case, the emission in the southern part of the disc would be contaminated by the southern subcore. Indeed, the ${ }^{13} \mathrm{CH}_{3} \mathrm{CN}$ PV plot (contours in Fig. 16) outlines three separate peaks, with the southernmost peak (indicated by the arrow in the figure) being clearly detached from the other two, both in space $\left(\sim 0^{\prime \prime} .2\right.$ or $1500 \mathrm{AU})$ and velocity $\left(\sim 5 \mathrm{~km} \mathrm{~s}^{-1}\right)$.

We conclude that the hypothesis of a Keplerian disc about a $20 M_{\odot}$ star in core A1, although speculative, cannot be disregarded. 


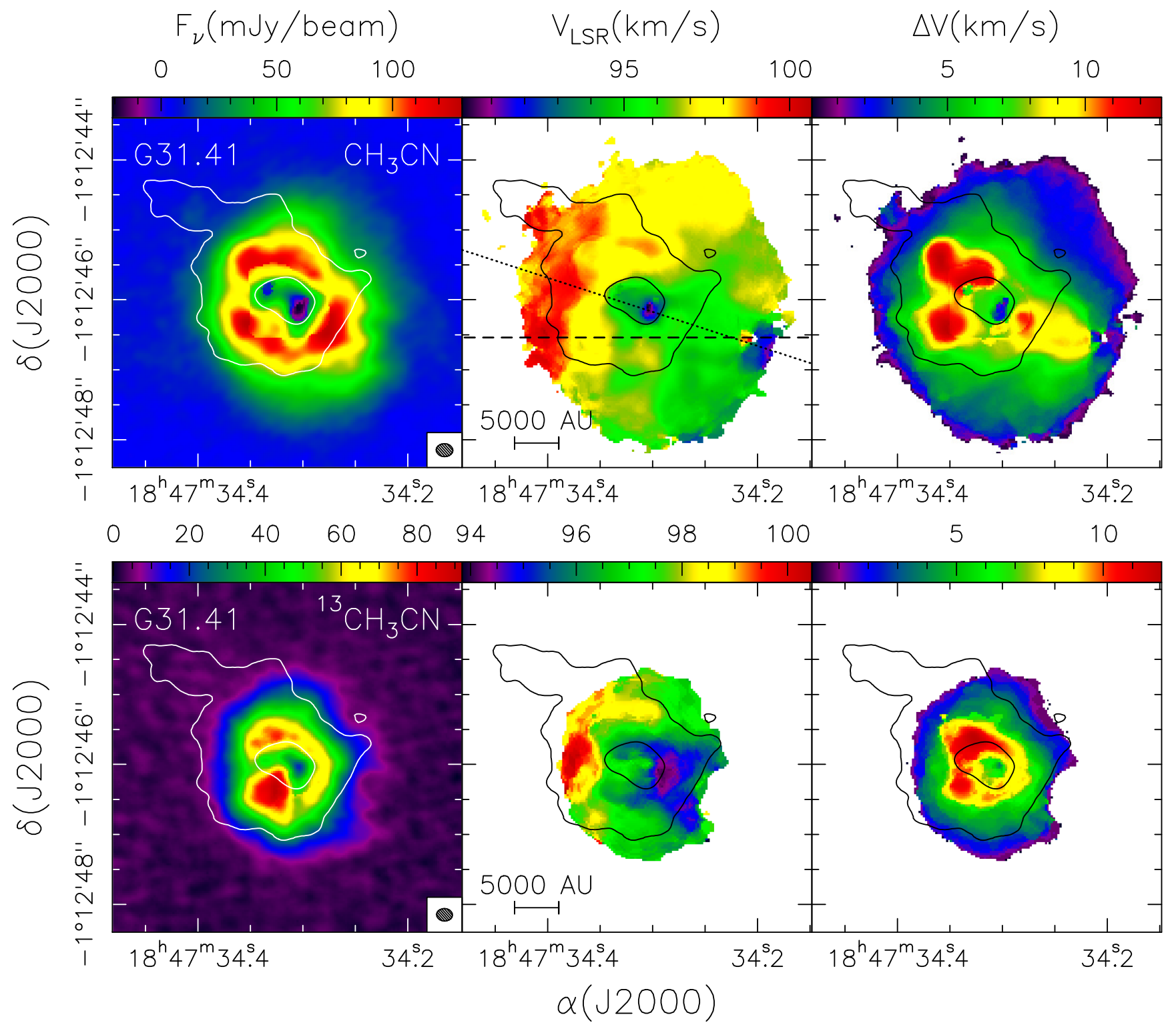

Fig. 9. Same as Fig. 7, but for G31.41. The dashed line marks the axis of the bipolar outflow detected in the present study (see Fig. 14). The dotted line denotes the cut adopted for the PV plots in Fig. 19.

\subsection{G29.96}

The HMC in this source lies very close to the apex of a wellknown cometary UC HII region, whose bright rim is clearly detected in our continuum map (Fig. 2). Figure 13 demonstrates that the velocity gradient observed in $\mathrm{CH}_{3} \mathrm{CN}$ is approximately perpendicular to the $\mathrm{SiO}$ bipolar outflow that emerges from the HMC itself. We thus consider the PV plot along a direction with $\mathrm{PA}=52^{\circ}$, passing through the HMC.

As can be seen in Fig. 17, this plot is roughly consistent with the butterfly-shaped emission expected for rotation about the outflow axis with $v_{\text {rot }} \propto R^{-\alpha}$. In particular, the white Keplerian pattern in the figure corresponds to a central mass of $10 M_{\odot}$. We note that this is shown only for the sake of comparison and is not a fit to the data. When comparing this pattern with the observed emission, one must take into account that the former assumes and edge-on geometry, zero line width, and infinite angular resolution. Moreover, it does not include a radial component of the velocity, due to infall, which is suggested by the inverse $\mathrm{P}$ Cygni profile detected in the ${ }^{13} \mathrm{CO}(2-1)$ line by Beltrán et al. (2011a). Multiplicity may also disturb the velocity field, as shown by Beuther et al. (2007), who resolved the main core into substructures. Therefore, a precise estimate of the stellar mass from the PV plot requires fitting the data with a disc model such as that by Johnston et al. (2015), which also takes into consideration the inclination of the disc with respect to the line of sight ${ }^{6}$.

\subsection{G31.41}

The literature about this HMC is very rich (see e.g. Cesaroni et al. 1994b, 2010; Moscadelli et al. 2013; Mayen-Gijon et al. 2014, and references therein). In particular, previous IRAM-PdBI (Beltrán et al. 2004, 2005) and SMA (Cesaroni et al. 2011) observations have confirmed the existence of a clear velocity gradient in $\mathrm{CH}_{3} \mathrm{CN}$ directed approximately $\mathrm{NE}-\mathrm{SW}$, but could not establish the presence of an outflow associated with the core. The $\mathrm{CO}(2-1)$ line maps by Cesaroni et al. (2011)

6 We stress that the dynamical mass, derived for the central star assuming an edge-on Keplerian disc, is a lower limit. 


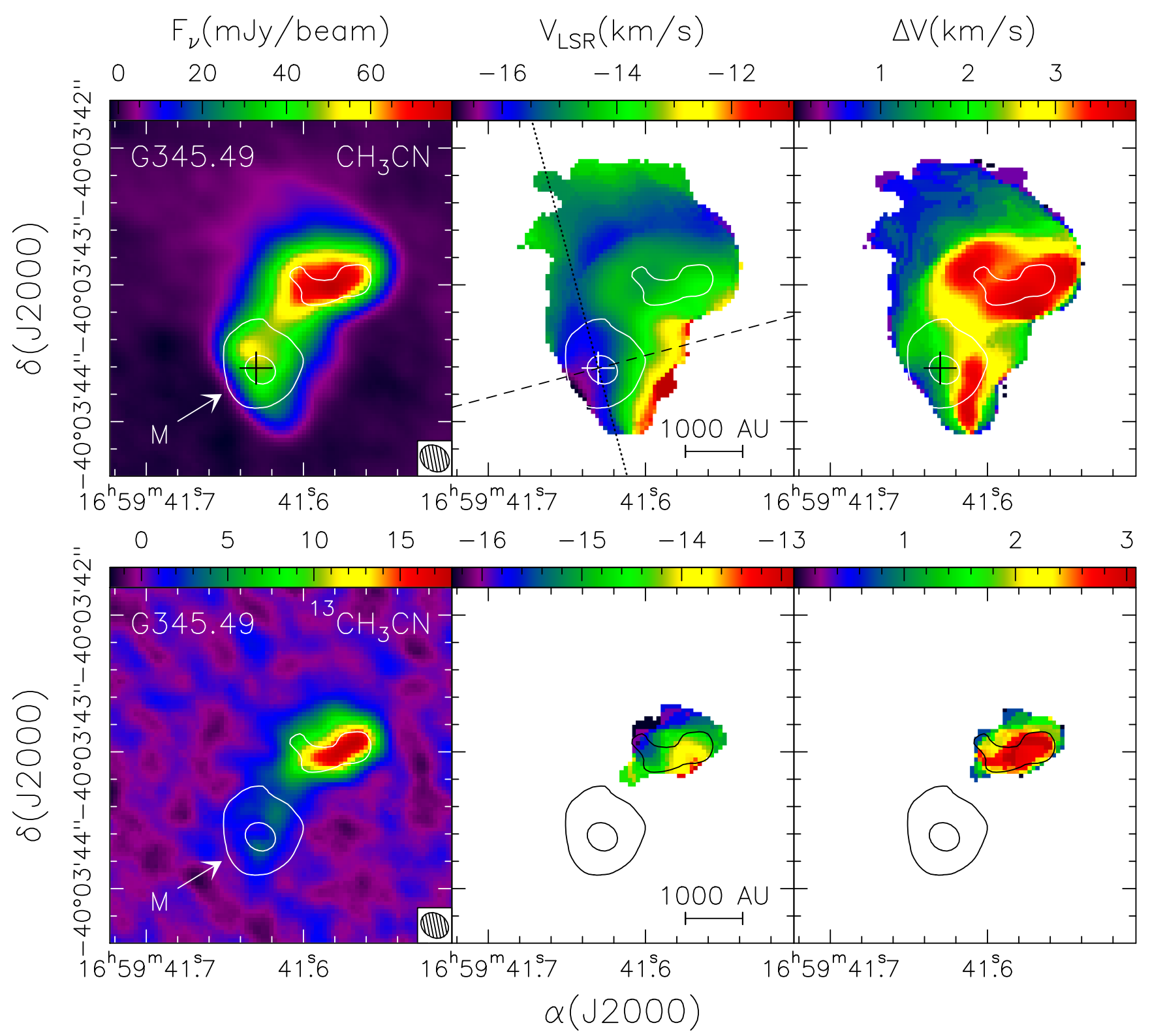

Fig. 10. Same as Fig. 7, but for G345.49. The cross marks the position of the central knot of the radio jet imaged by Guzmán et al. (2010), while the dashed line denotes the radio jet axis. Label M marks the core of interest for our study, as in Fig. 2.

suggested the existence of a bipolar symmetry in the E-W direction roughly centred on the HMC, but the confusion with emission from the envelope and perhaps from unassociated gas along the line of sight made it difficult to draw any conclusions.

The new ALMA data represent an increase by a factor of $\sim 4$ in angular resolution and provide us with a more selective jet/outflow tracer (i.e. SiO). The main findings can be summarized as follows:

- Albeit well resolved, the continuum emission unveils a very smooth structure of the HMC without any hint of fragmentation (Fig. 2). However, such a simple structure does not necessarily mirror the real structure of the core because the continuum emission at $1.4 \mathrm{~mm}$ is in all likelihood optically thick, as suggested by the high value of the peak brightness temperature $(\sim 130 \mathrm{~K})$, the highest in our sample. Such a high opacity/brightness explains the existence of deep absorption even in $\mathrm{CH}_{3} \mathrm{CN}$ lines with excitation as high as $\sim 200 \mathrm{~K}$ (see
Fig. 18), which in turn is responsible for the dip at the centre of the ring-like maps in the left panels of Fig. 9.

- The velocity of the gas increases smoothly from SW to NE (Fig. 9). However, the study of the velocity field on a small scale is hindered by the large continuum opacity close to the core centre. This prevents us from inspecting the kinematics of the central region, where any putative sign of Keplerianlike rotation could be detected.

- All $\mathrm{CH}_{3} \mathrm{CN}$ lines (and many others) present inverse P Cygni profiles (see Fig. 18) towards the core centre, a strong indication of infall. This is why the velocity at the centre of the first moment map in Fig. 9 appears blue shifted. The presence of infall is relevant to the existence of a disc in that it could affect its structure and stability, depending on the ratio between the rotation period and accretion time scale (see Fig. 14 in Beltrán \& de Wit 2016).

- A clear bipolar outflow is detected in the $\mathrm{SiO}(5-4)$ line (Fig. 14). The blue and red lobes lie on the same side as 
R. Cesaroni et al.: Chasing discs around O-type (proto)stars: Evidence from ALMA observations
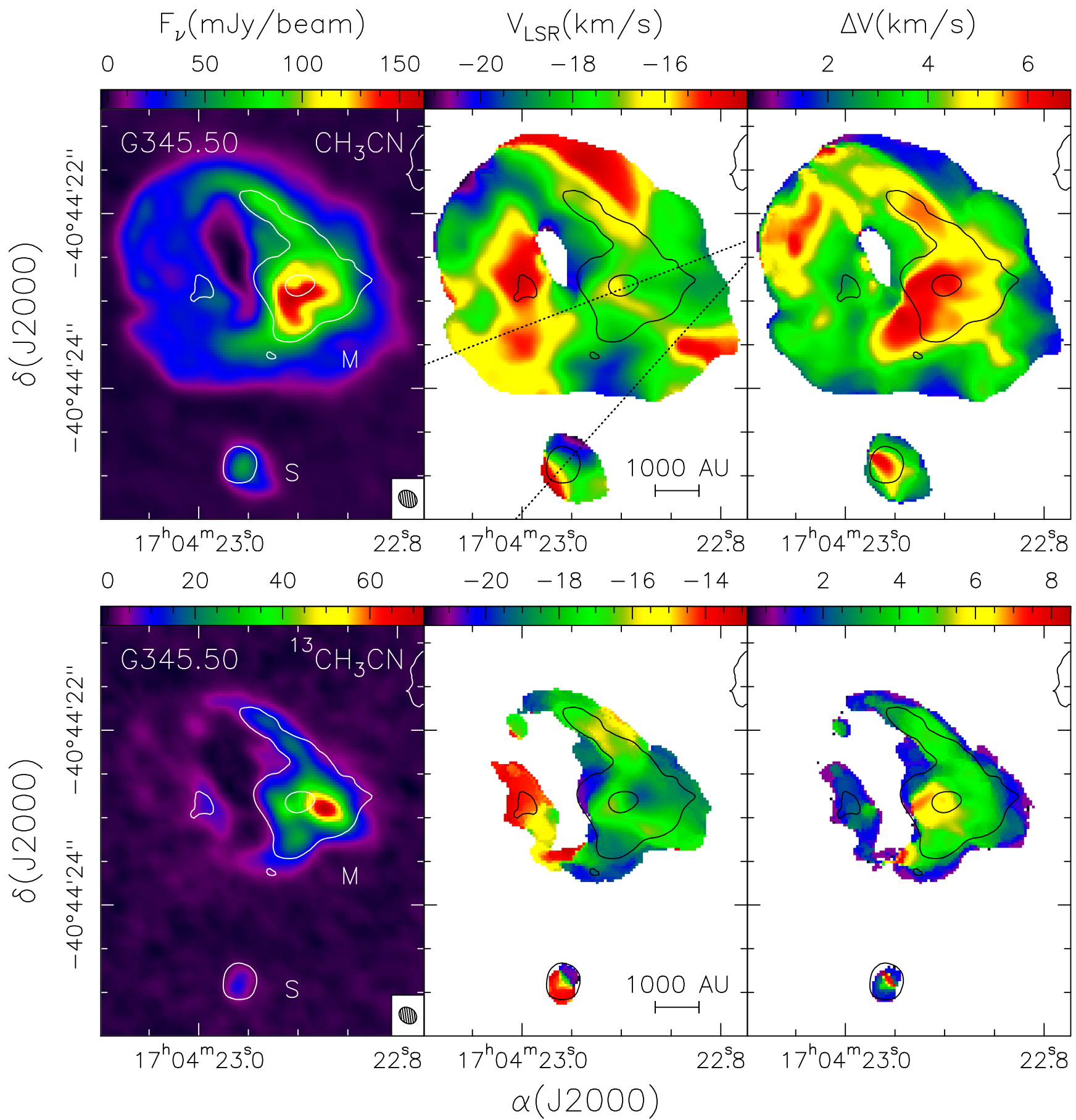

Fig. 11. Same as Fig. 7, but for G345.50. Labels M and S mark the corresponding cores, as in Fig. 2. The dotted lines denote the cuts adopted for the PV plots in Figs. 22 and 23.

the $\mathrm{CH}_{3} \mathrm{CN}$ blue- and red-shifted emission. However, the direction of the $\mathrm{CH}_{3} \mathrm{CN}$ velocity gradient $\left(\mathrm{PA} \simeq 68^{\circ}\right)$ is significantly different from that of the outflow axis $\left(\mathrm{PA} \simeq 0^{\circ}\right.$; see Fig. 9) and the geometrical centre of the outflow is offset by $\sim 0$ '. 65 to the south with respect to the HMC centre (see Figs. 9 and 14). Therefore, we do not believe that the $\mathrm{SiO}$ outflow and the $\mathrm{CH}_{3} \mathrm{CN}$ velocity gradient are due to the same phenomenon and associated with the same source.

With this in mind, we study the PV plot along the direction with $\mathrm{PA}=68^{\circ}$ passing through the HMC. In order to minimize the effect of the absorption towards the core centre, we average the emission along the direction perpendicular to the cut of the PV plot. Moreover, since the $\mathrm{CH}_{3} \mathrm{CN}$ lines are heavily affected by the absorption, we prefer to use the isotopologue ${ }^{13} \mathrm{CH}_{3} \mathrm{CN}$.

We compare the PV plot of the ${ }^{13} \mathrm{CH}_{3} \mathrm{CN}(13-12) K=2$ transition in Fig. 19a, with the same plot of the $\mathrm{CH}_{3} \mathrm{CN}(12-11)$ $v_{8}=1 K, l=6,1$ line in Fig. 19b. The striking result is that the slope of the emission is steeper in the $v_{8}=1$ line. Since the gas temperature is expected to increase towards the core centre and the $v_{8}=1$ transition has a greater excitation energy $(778 \mathrm{~K})$ than the ground state line $(96 \mathrm{~K})$, the different slopes indicate that at smaller radii the gas velocity increases. In other words, the higher the excitation of a line, the closer to the core centre the line will be observed, and Fig. 19 shows that the gas also 


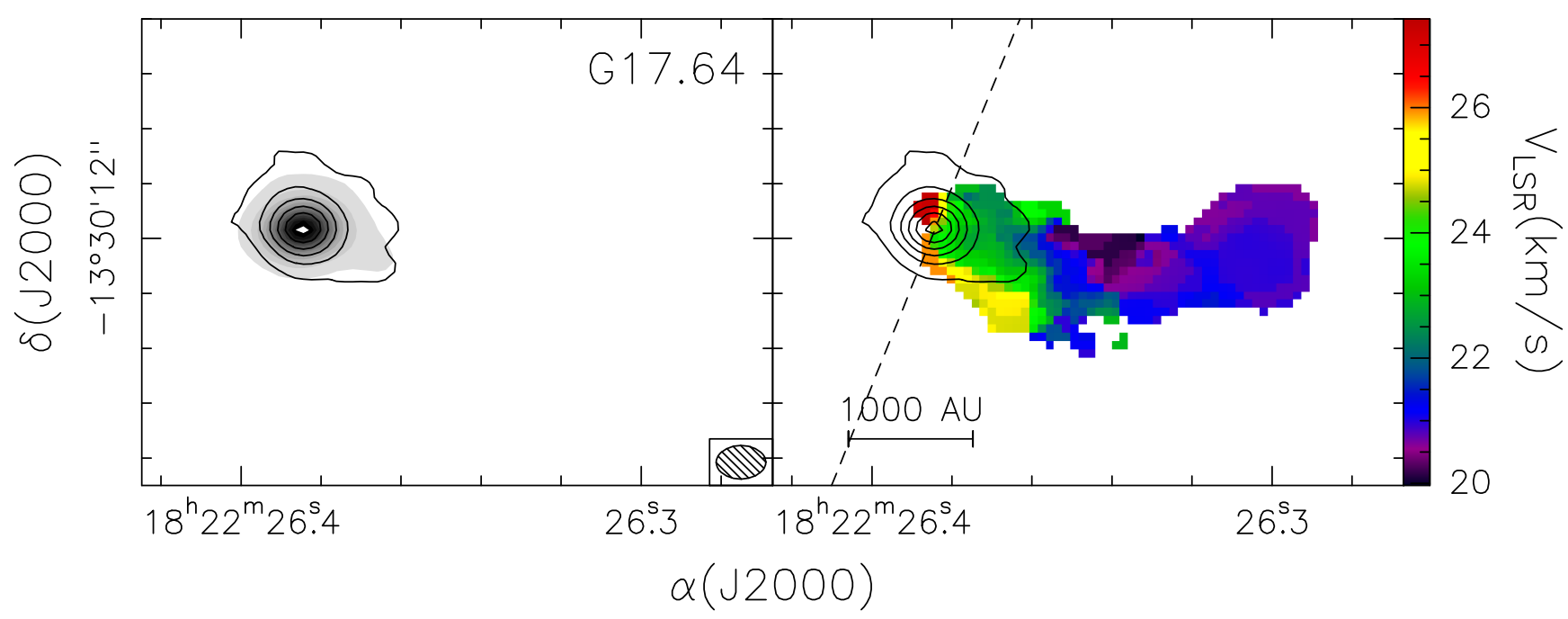

Fig. 12. Left: contour map obtained by averaging the emission over the $\mathrm{SiO}(5-4)$ line from 4.0 to $39.1 \mathrm{~km} \mathrm{~s}^{-1}$, overlaid on the map (grey scale) of the $1.4 \mathrm{~mm}$ continuum emission in G17.64. Contour levels range from 2.8 to $19.8 \mathrm{in}$ steps of $3.4 \mathrm{mJy} / \mathrm{beam}$. The synthesized beam is shown in the bottom right. Right: same as left panel, overlaid on the first moment map of the $\mathrm{CH}_{3} \mathrm{CN}(12-11) K=2$ line. The dashed line indicates the direction of the axis of the ${ }^{12} \mathrm{CO}$ outflow detected by Kastner et al. (1994).

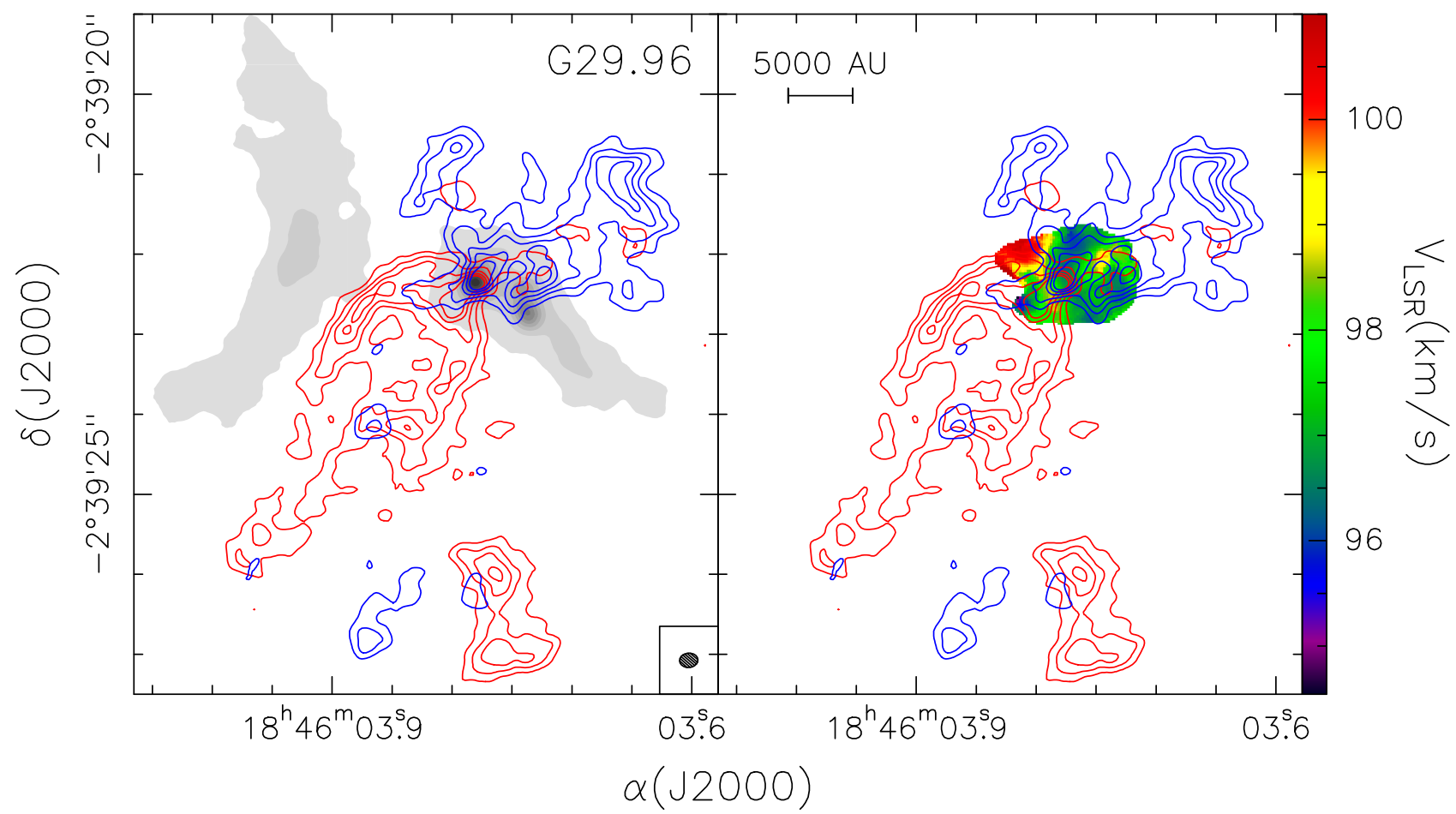

Fig. 13. Left: contour maps of the blue- and red-shifted $\mathrm{SiO}(5-4)$ line emission overlaid on the map (grey scale) of the $1.4 \mathrm{~mm}$ continuum emission in G29.96. The $\mathrm{SiO}$ emission has been integrated over the velocity intervals 83.5 to $91.6 \mathrm{~km} \mathrm{~s}^{-1}$ and 102.4 to $110.5 \mathrm{~km} \mathrm{~s}^{-1}$ for the blue and red wings, respectively. Contour levels range from 6 to 26 in steps of $5 \mathrm{mJy} /$ beam. The synthesized beam is shown in the bottom right. Right: same as left panel, overlaid on the first moment map of the $\mathrm{CH}_{3} \mathrm{CN}(12-11) \mathrm{K}=2$ line.

has higher velocity closer to the centre. This result is consistent with $v_{\text {rot }} \propto R^{-\alpha}$. Indeed, the same type of "steepening" of the PV plot with increasing energy of the transition can be seen in Fig. 4 in Johnston et al. (2015), where the observed PV plots are fitted with a disc undergoing Keplerian rotation.

With all the caveats previously discussed and related to the large continuum opacity and presence of absorption, we believe that our findings are consistent with a scenario where the gas in G31.41 is rotating and infalling towards the core centre with conservation of angular momentum, and eventually accreting onto one or more stars through a circumstellar disc.

\section{5. $G 345.49$}

Although several cores are present within the primary beam of our ALMA observations, we have chosen to focus on the strongest continuum source, $\mathrm{M}$, which lies at the centre of the thermal radio jet observed by Guzmán et al. (2010, 2016). The central radio source and the direction of the jet axis are labelled in Fig. 10 with a cross and a dashed line, respectively. Comparison between the jet direction and the first moment map indicates that $\mathrm{CH}_{3} \mathrm{CN}$ is tracing expansion along the jet. However, the velocity gradient is not exactly directed along the jet axis, 
R. Cesaroni et al.: Chasing discs around O-type (proto)stars: Evidence from ALMA observations

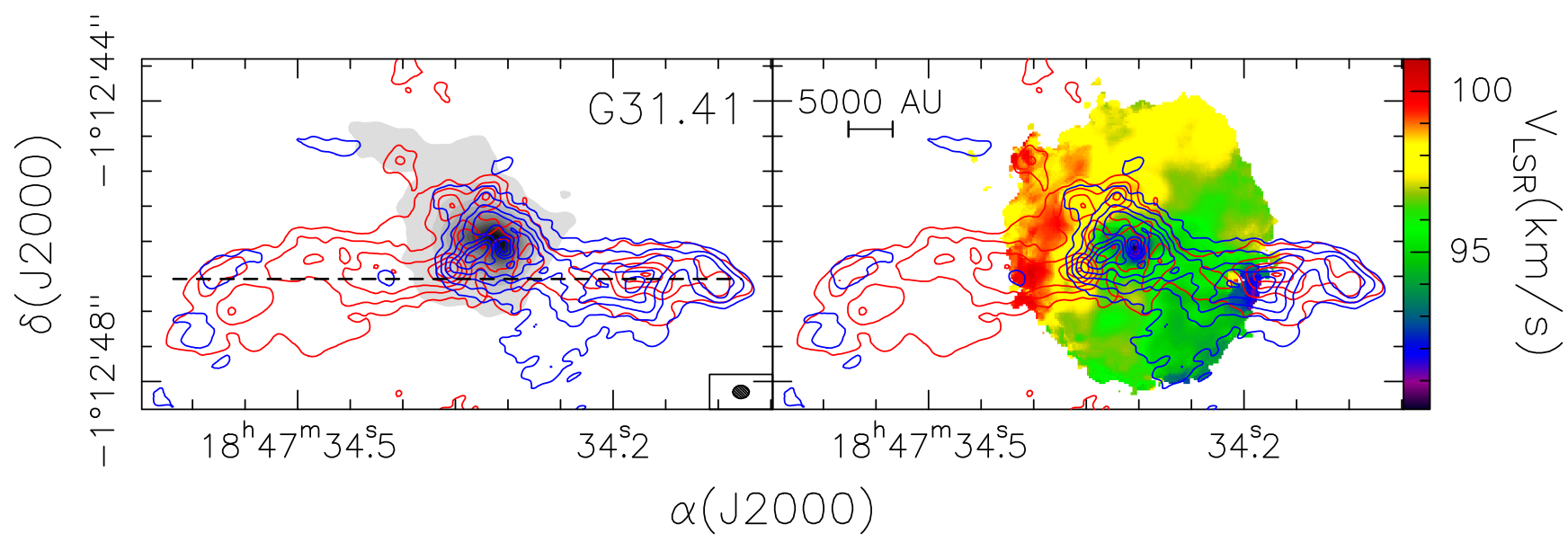

Fig. 14. Same as Fig. 13, but for G31.41. The dashed line marks the axis of the SiO bipolar outflow. The SiO emission has been integrated over the velocity intervals 76.1 to $95.0 \mathrm{~km} \mathrm{~s}^{-1}$ and 100.4 to $119.2 \mathrm{~km} \mathrm{~s}^{-1}$ for the blue and red wings, respectively. Contour levels range from 7.2 to 37.2 in steps of $6 \mathrm{mJy} / \mathrm{beam}$.

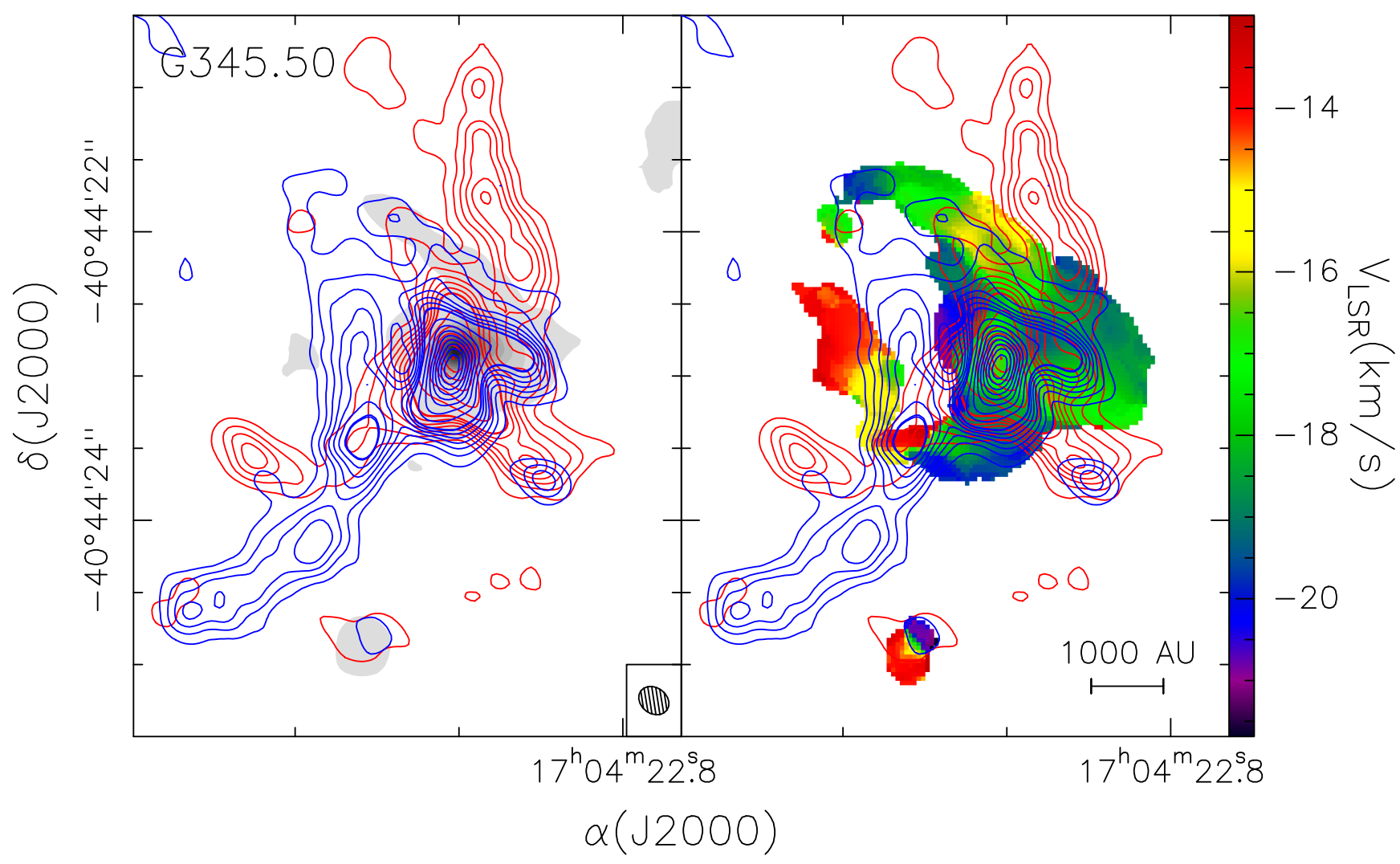

Fig. 15. Same as Fig. 13, but for G345.50. The image in the right panel is the first moment of the ${ }^{13} \mathrm{CH}_{3} \mathrm{CN}(13-12) K=2$ line. The SiO emission has been integrated over the velocity intervals -23.8 to $-18.4 \mathrm{~km} \mathrm{~s}^{-1}$ and -15.7 to $-10.3 \mathrm{~km} \mathrm{~s}^{-1}$ for the blue and red wings, respectively. Contour levels range from 4.2 to 29.4 in steps of $4.2 \mathrm{mJy} /$ beam and from 30 to $130.8 \mathrm{in} \mathrm{steps} \mathrm{of} 12.6 \mathrm{mJy} / \mathrm{beam}$.

but appears to have a component in the perpendicular direction as well. The PV plot in Fig. 20 was obtained along the direction with $\mathrm{PA}=+15^{\circ}$, i.e. perpendicular to the jet axis, through the continuum peak. In order to maximize the signal-to-noise, the emission was been averaged over a stripe spanning \pm 0 '.25 across the cut. We note that the corresponding PV plot for the ${ }^{13} \mathrm{CH}_{3} \mathrm{CN}(13-12)$ line cannot be used, because the emission is barely detected (see Fig. 10).

The $\mathrm{CH}_{3} \mathrm{CN}$ emission in Fig. 20 appears to trace only half of the butterfly-shaped pattern expected for Keplerian rotation about a $10 M_{\odot}$ star, as the red-shifted "wing" is missing. Such an incomplete structure is not surprising as the presence of a strong free-free source suggests that the onset of a hyper-compact HII region at the jet centre could have deeply perturbed the disc morphology. In addition, significant deviations from axial symmetry are indeed predicted by numerical simulations (Krumholz et al. 2007b; Peters et al. 2010, 2011; Kuiper et al. 2011; Klassen et al. 2016).

\subsection{G345.50}

This source lies at the centre of a bipolar nebula oriented approximately NE-SW, which can be seen at IR wavelengths in 


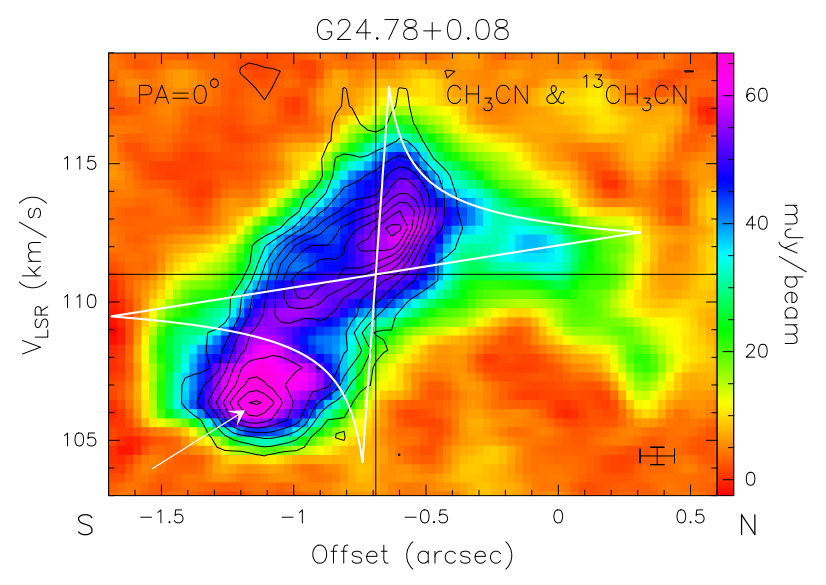

Fig. 16. Position-velocity plots of the $\mathrm{CH}_{3} \mathrm{CN}(12-11) \mathrm{K}=2$ (colour image) and ${ }^{13} \mathrm{CH}_{3} \mathrm{CN}(13-12) K=2$ (contours) lines obtained along a N-S cut across the hypercompact HII region lying inside the HMC G24.78 A1 (see Beltrán et al. 2007). The offset is measured with respect to the phase centre (given in Table 1). The white pattern outlines the region inside which emission is expected if the gas is undergoing Keplerian rotation about a $20 M_{\odot}$ star (the mass of the $\mathrm{O} 9.5$ star ionizing the hypercompact HII region; see Beltrán et al. 2007), assuming edgeon inclination. The horizontal and vertical lines mark, respectively, the position of the HII region and the systemic LSR velocity. The cross in the bottom right indicates the angular and spectral resolution. The arrow indicates the emission peak probably associated with a secondary subcore.

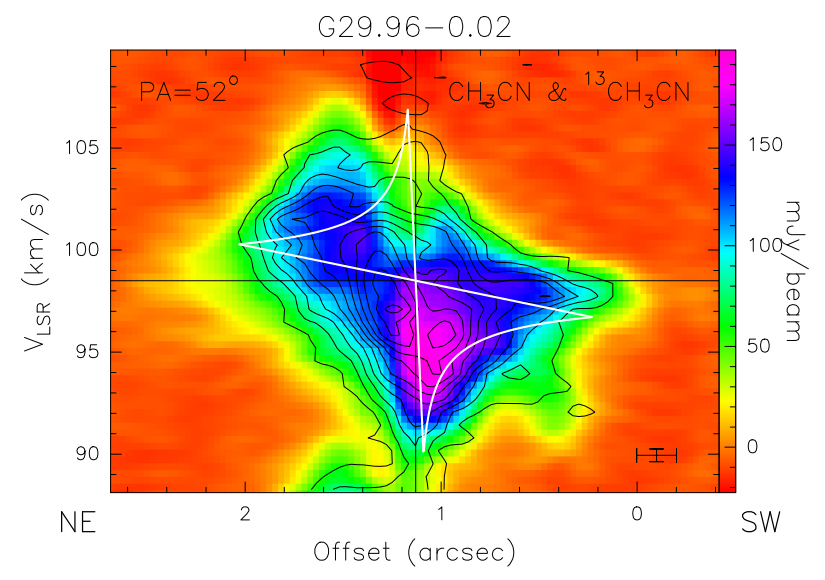

Fig. 17. Same as Fig. 16, but for the HMC in G29.96. The cut is made through the $\mathrm{HMC}$ along $\mathrm{PA}=52^{\circ}$ (dotted line in Fig. 8). The white pattern corresponds to Keplerian rotation about a $10 M_{\odot}$ star.

the Spitzer/GLIMPSE images (Churchwell et al. 2009). While the focus of the observations was on the main core at the centre of the field covered by ALMA, a southern core was serendipitously detected. We will refer to the main and southern cores, respectively, as G345.50 M and G345.50 S (see Fig. 2).

Both cores are detected in the $\mathrm{CH}_{3} \mathrm{CN}$ and ${ }^{13} \mathrm{CH}_{3} \mathrm{CN}$ lines, which trace clear velocity gradients directed roughly E-W (for $\mathrm{G} 345.50 \mathrm{M}$ ) and $\mathrm{N}-\mathrm{S}$ (for G345.50 S). This can be seen in Fig. 11 and even more clearly for source $M$ in Fig. 21, where the first moment map has been clipped outside the border of the core, namely outside the $5 \sigma$ contour level of the continuum emission. Further analysis of the velocity field is presented in Figs. 22 and 23, where we show the PV plots of the $\mathrm{CH}_{3} \mathrm{CN}(12-$ 11) $K=2$ line along suitable directions across the two cores. As was done for the other sources, we show the pattern expected for Keplerian rotation about a $10 M_{\odot}$ star. Remarkably, both plots

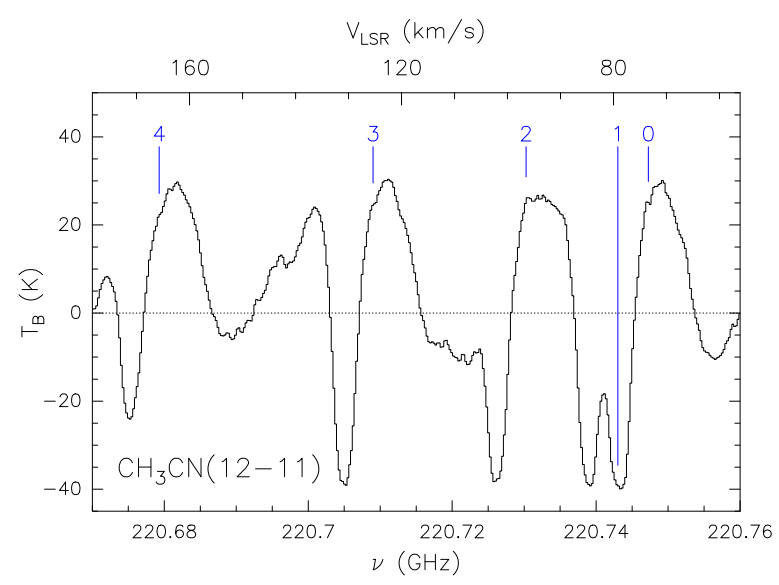

Fig. 18. Spectrum of the $\mathrm{CH}_{3} \mathrm{CN}(12-11) K=2$ to 4 lines towards the absorption dip of the G31.41 HMC. The vertical lines mark the different $K$ components. The LSR velocity given in the upper $x$-axis refers to the $K=2$ component. All lines present inverse P Cygni profiles, except the $K=1$ component because this is blended with the $K=0$.

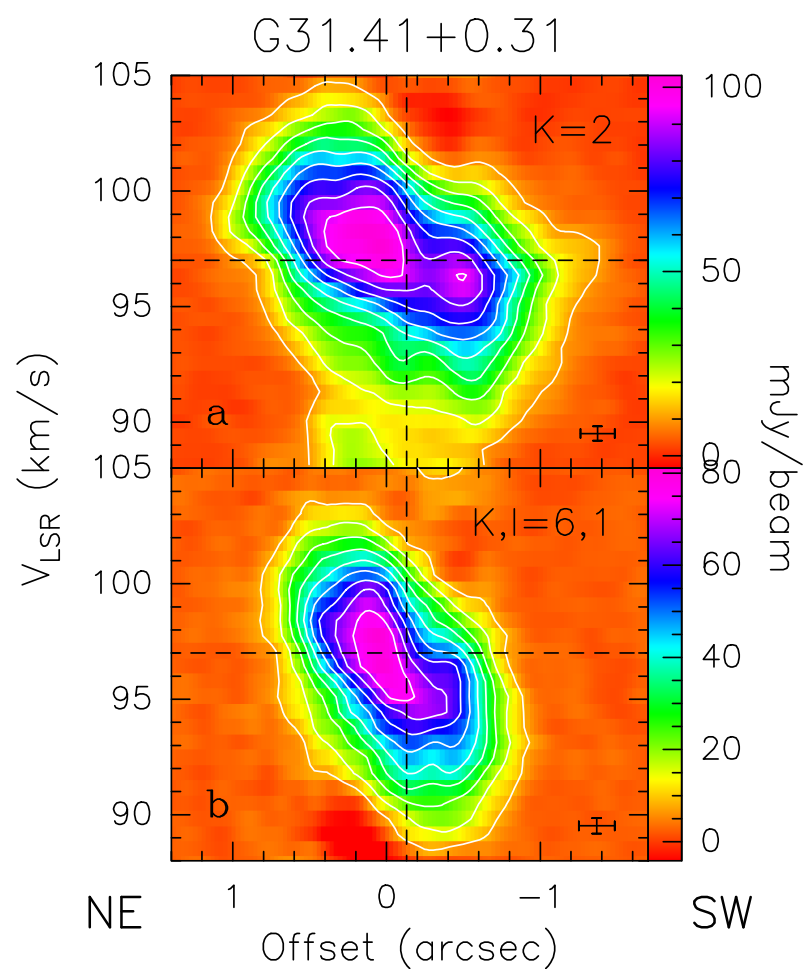

Fig. 19. a) $\mathrm{PV}$ plot of the ${ }^{13} \mathrm{CH}_{3} \mathrm{CN}(13-12) \mathrm{K}=2$ line emission (excitation energy of $96 \mathrm{~K}$ ) in $\mathrm{G} 31.41$ along a direction with $\mathrm{PA}=68^{\circ}$ passing through the HMC (dotted line in Fig. 9). The vertical and horizontal dashed lines indicate, respectively, the position of the continuum peak and the systemic velocity. The cross in the bottom right corner denotes the angular and spectral resolutions. b) Same as top panel, but for the $\mathrm{CH}_{3} \mathrm{CN}(12-11) v_{8}=1 \mathrm{~K}, l=6,1$ line (excitation energy of $778 \mathrm{~K}$ ).

present a butterfly shape, consistent with an increase in the rotation velocity towards the centre. As already pointed out, an estimate of the central point mass is hazardous without a model fit. However, the main difference between the two PV plots is mostly due to $\mathrm{G} 345.50 \mathrm{M}$ having a more irregular shape, whereas the range in velocity is basically the same as for G345.50 S.

One may speculate that the mass of the two stars is basically the same and the different shape of the PV plots could depend on the surrounding environment, with G345.50 S being 
R. Cesaroni et al.: Chasing discs around O-type (proto)stars: Evidence from ALMA observations

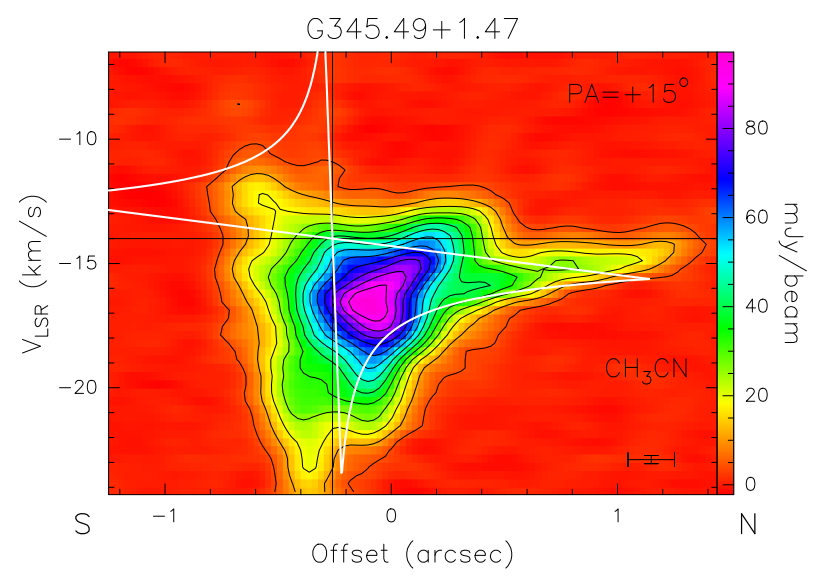

Fig. 20. $\mathrm{PV}$ plot of the $\mathrm{CH}_{3} \mathrm{CN}(12-11) \mathrm{K}=2$ line emission (colour scale and contours) for G345.49 M. The cut is made through the strongest continuum peak, along $\mathrm{PA}=15^{\circ}$. Unlike the $\mathrm{PV}$ plots of the other sources, here we do not plot the ${ }^{13} \mathrm{CH}_{3} \mathrm{CN}(13-12)$ emission since it is barely detected (see Fig. 10). The white pattern corresponds to Keplerian rotation about a $10 M_{\odot}$ star.

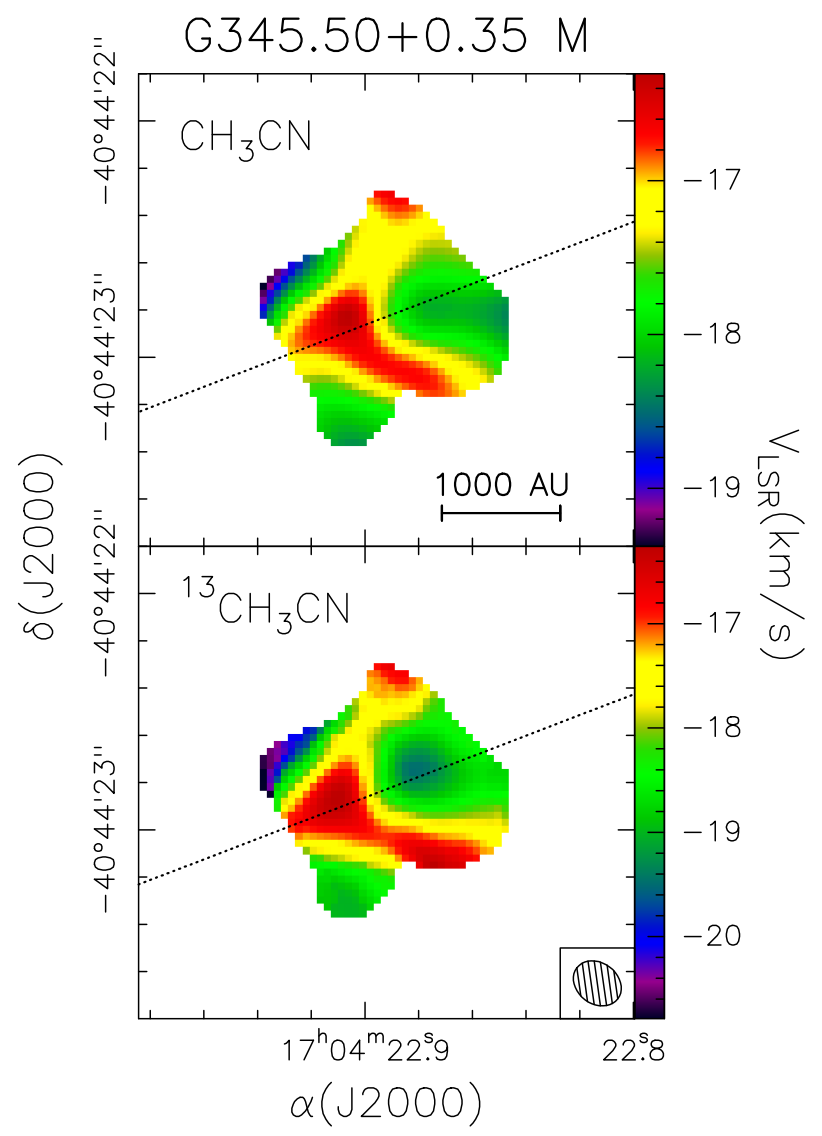

Fig. 21. First moment map of the $\mathrm{CH}_{3} \mathrm{CN}(12-11) K=2$ (top panel) and ${ }^{13} \mathrm{CH}_{3} \mathrm{CN}(13-12) K=2$ (bottom) lines towards the HMC in $\mathrm{G} 345.50 \mathrm{M}$. The data have been clipped outside the $5 \sigma$ contour level of the continuum emission to show the velocity gradient along the direction denoted by the dotted line. The synthesized beam is shown in the bottom right corner.

more isolated and hence less perturbed, for instance by interactions with a (deeply embedded) stellar cluster. In this scenario, G345.50 M would be closer to the centre of the gravitational potential well and thus could have evolved faster, warming up the surrounding material. The combination of infall and outflow

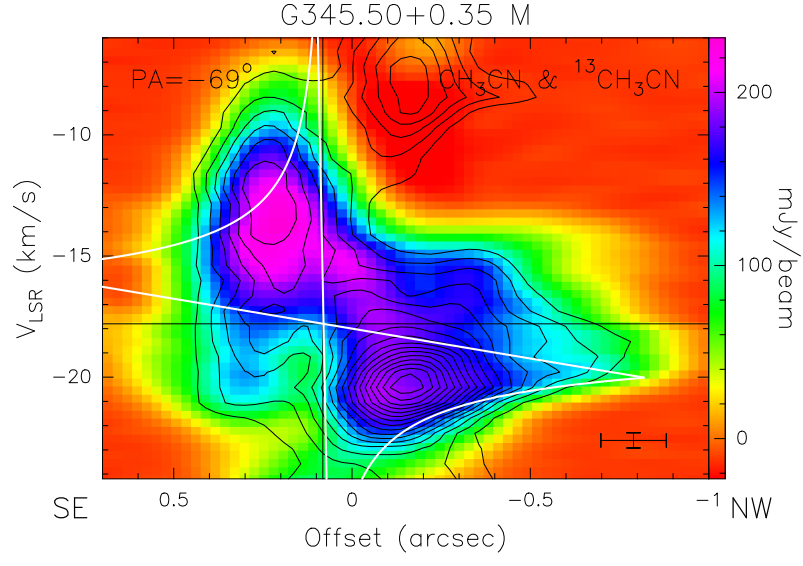

Fig. 22. Same as Fig. 17, but for the HMC in G345.50 M. The cut is made through the HMC along PA $=-69^{\circ}$ (dotted line in Fig. 11). The peak at $-00^{\prime \prime} 15$ and $-8 \mathrm{~km} \mathrm{~s}^{-1}$ of the contour map is due to emission in an unidentified molecular line.

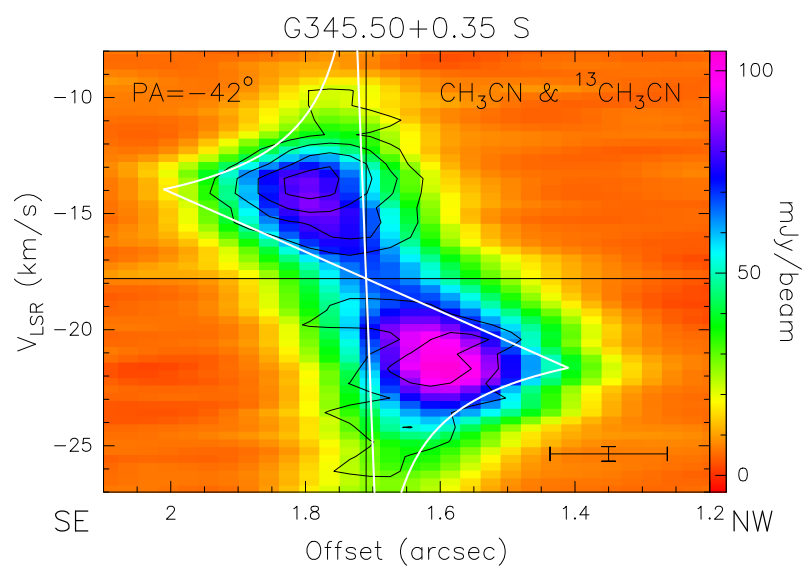

Fig. 23. Same as Fig. 17, but for the HMC in G345.50 S. The cut is made through the $\mathrm{HMC}$ along $\mathrm{PA}=-42^{\circ}$ (dotted line in Fig. 11).

could be the reason that the structure is so complex. Vice versa, $\mathrm{S}$ would have formed later and/or have a lower accretion rate with less impact on its surroundings, which are less complex owing to simpler dynamics.

The morphology of the $\mathrm{SiO}(5-4)$ line emission appears much more complex than in other sources (see Fig. 15). The blue- and red-shifted emissions outline two elongated structures on opposite sides with respect to $\mathrm{G} 345.50 \mathrm{M}$, but no obvious symmetry axis can be identified. In the case of $\mathrm{G} 345.50 \mathrm{~S}$, the $\mathrm{SiO}$ emission is very faint and no clear pattern can be recognized. It is thus difficult to establish whether a bipolar outflow (possibly related to the large-scale bipolar nebula seen at IR wavelengths) is present in this field. It must be noted, however, that the location of the blue-shifted gas on the eastern side coincides with the IR reflection nebula, consistent with seeing more scattered light from the lobe facing the observer. In contrast, most of the IR nebulosity on the western side is due to the extended cometary HII region imaged in the radio by Garay et al. (2006), and is hence not tracing the red lobe of the (putative) outflow.

\section{Are there discs around O-type stars?}

As previously explained, the goal of our project was to establish whether circumstellar discs are present around O-type (proto)stars. Has our search been successful? Based on the results discussed in the previous sections, we can state that we find 


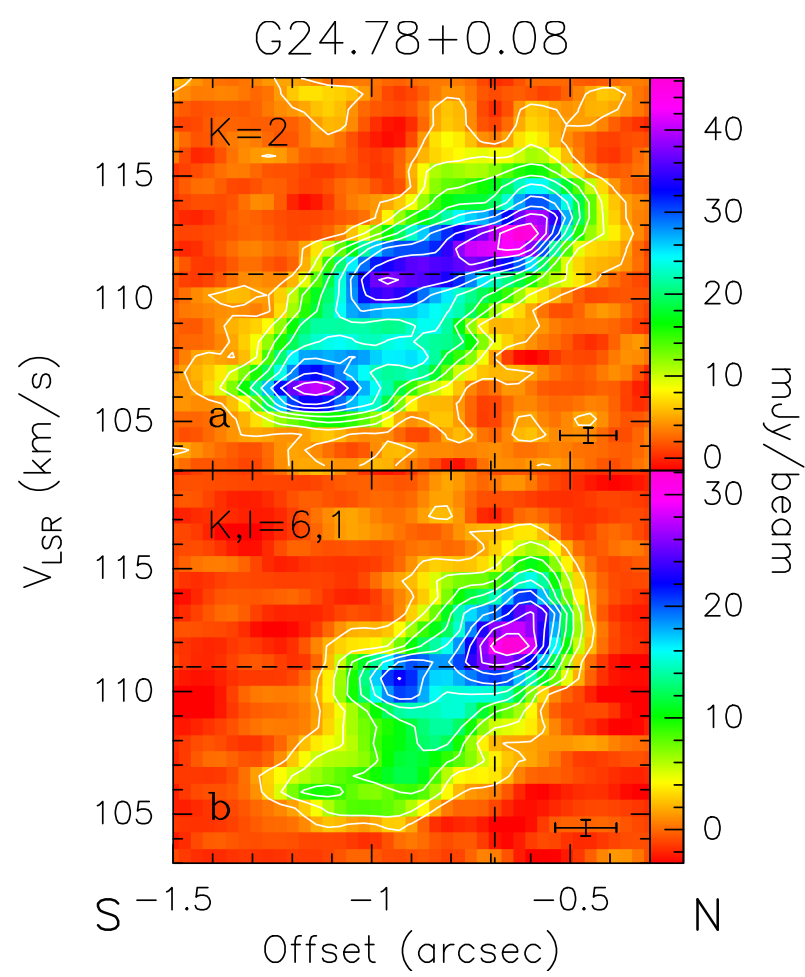

Fig. 24. Same as Fig. 19, but for the HMC in G24.78 A1.

evidence for rotation in at least three cases (G29.96, G345.50 M, G345.50 S), plus three additional, albeit more questionable, candidates (G24.78, G31.41, G345.49). This evidence is based on the existence of velocity gradients across molecular cores. We have discussed the interpretation of these gradients in terms of rotation. While the fingerprint of Keplerian-like rotation is the butterfly shape of the PV plots (see Sect. 5), it is also useful to compare the PV plots of lines with different excitation energies, as was done for G31.41 (see Fig. 19). Since the temperature is expected to be higher close to the core centre where the gas rotates faster (because $v_{\text {rot }} \propto R^{-\alpha}$ ), then for increasing line energy the emission peak in the PV plot should move towards more extreme blue- and red-shifted velocities (see Sect. 5.4).

On the analogy of the analysis of G31.41, we thus compare the PV plot of the ${ }^{13} \mathrm{CH}_{3} \mathrm{CN}(13-12) \mathrm{K}=2$ line with that of a vibrationally excited line for all other cores where velocity gradients have been detected. Only for source G345.49 was the $\mathrm{CH}_{3} \mathrm{CN}$ line used instead of the ${ }^{13} \mathrm{CH}_{3} \mathrm{CN}$ transition, because the latter is undetected towards the core (see Fig. 10). The results are shown in Figs. 24-28. Although less remarkable than for G31.41, a significant difference is seen between the PV plots in the two lines, for G29.96, G345.50 M, and G345.50 S. In fact, dividing the PV plot in the bottom panel by that in the top panel, we find that in these objects the highest ratio between the vibrationally excited line and the ground state line is found close to the continuum peak and at relatively large velocity offsets, as expected for rotation speed-up towards the centre (see the discussion on G31.41 in Sect. 5.4). In particular, in G29.96 the maximum ratio is found at 0 ".9 and $91.5 \mathrm{~km} \mathrm{~s}^{-1}$, in $\mathrm{G} 345.50 \mathrm{M}$ at -0 ". 1 and $-23.3 \mathrm{~km} \mathrm{~s}^{-1}$, and in $\mathrm{G} 345.50 \mathrm{~S}$ at 1 '.' $6-25 \mathrm{~km} \mathrm{~s}^{-1}$. These results are consistent with the emission extending to higher velocities in the higher excitation line. While other interpretations are possible, these findings are consistent with these three sources undergoing Keplerian-like rotation.

Table 4 summarizes our conclusions for the different targets, whose distance, spatial resolution, and $L_{\mathrm{bol}} / M_{\mathrm{gas}}$ ratio are

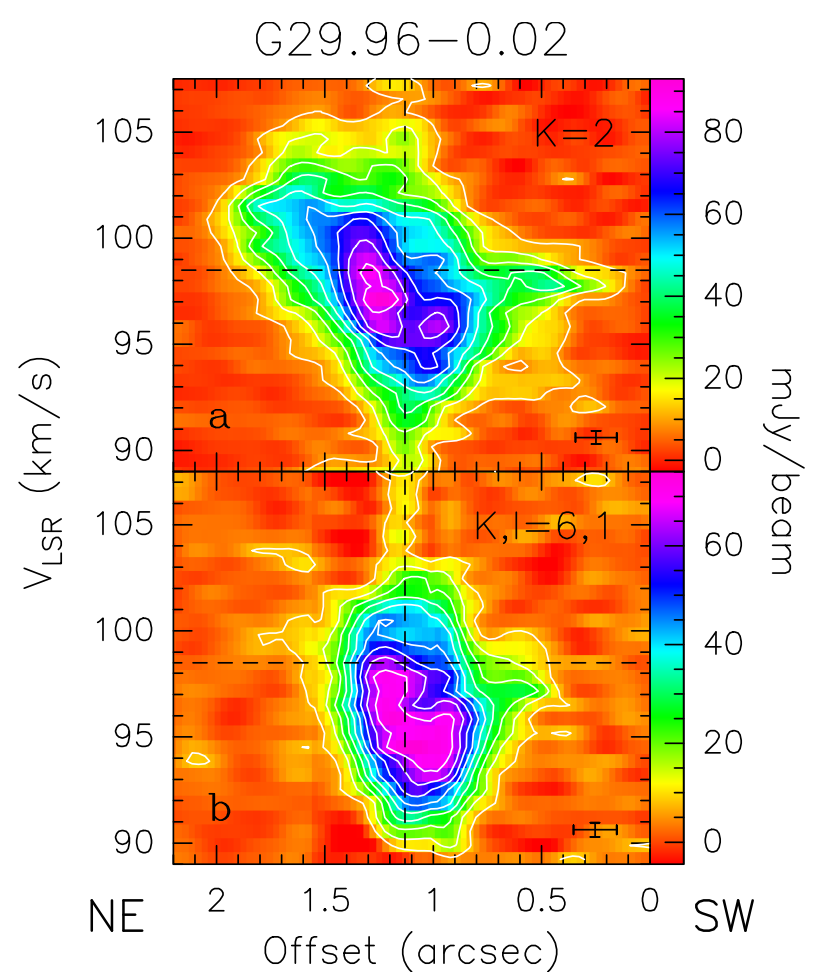

Fig. 25. Same as Fig. 19, but for the HMC in G29.96.

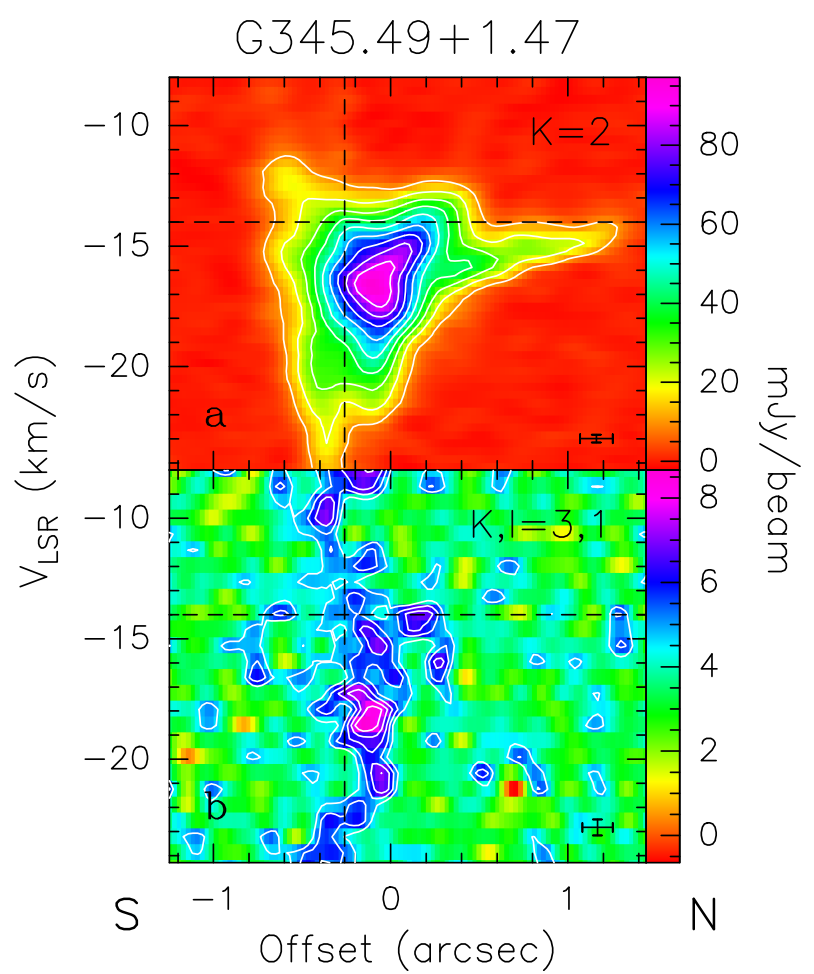

Fig. 26. Same as Fig. 19, but for the G345.49 $\mathrm{M}$ and the $\mathrm{CH}_{3} \mathrm{CN}(12-11)$ $K=2$ and $v_{8}=1 K, l=3,1$ lines.

also given. For the sake of comparison, we have also included the AFGL 4176 disc, detected by Johnston et al. (2015), the only bona fide disc around an O-type star known to date. We find some evidence consistent with the presence of a disc in all sources but G17.64. We note that we refer to the molecular component of the disc, whose absence does not rule out the presence 
R. Cesaroni et al.: Chasing discs around O-type (proto)stars: Evidence from ALMA observations

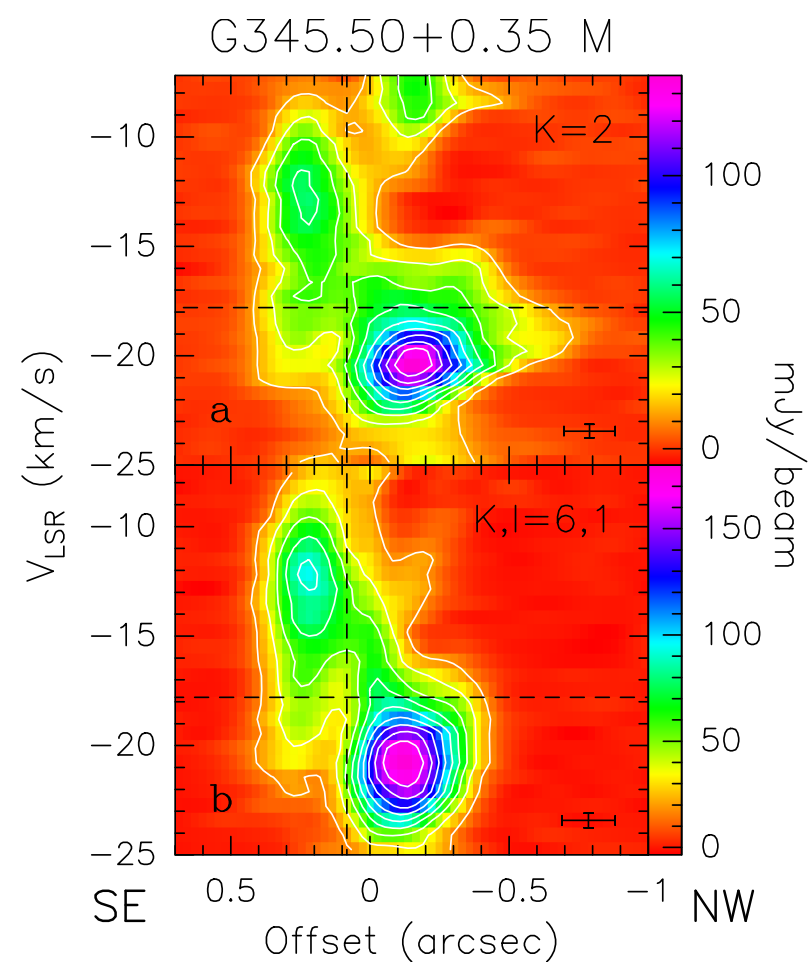

Fig. 27. Same as Fig. 19, but for G345.50 M.

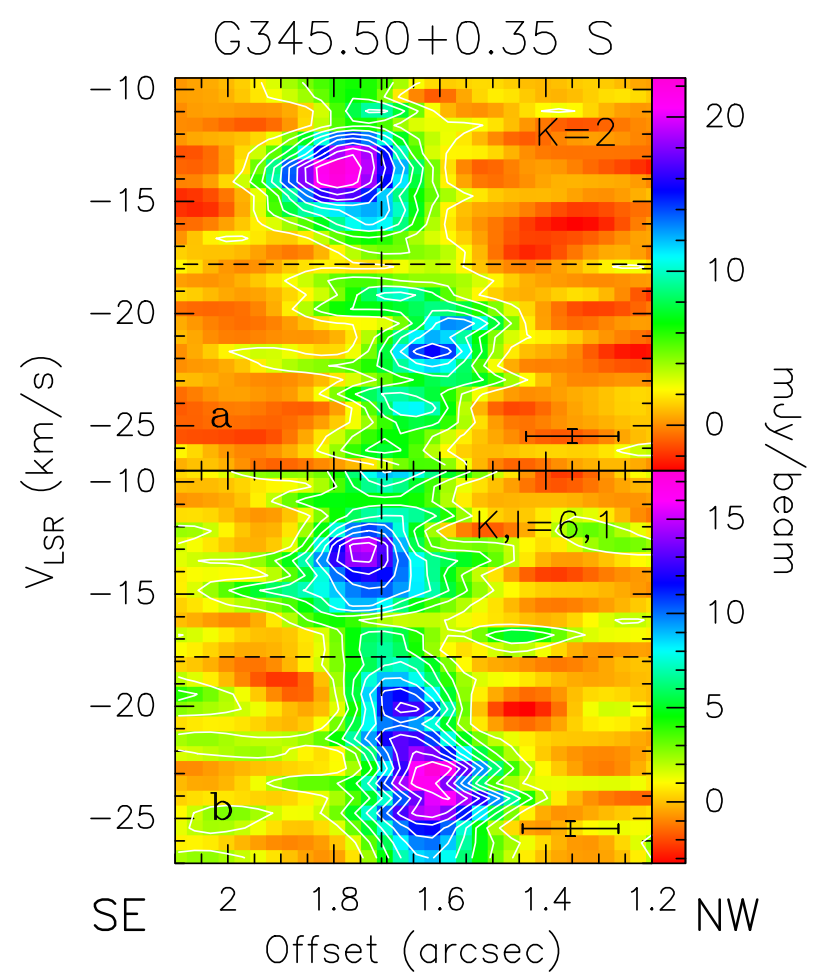

Fig. 28. Same as Fig. 19, but for G345.50 S.

of an ionized disc or any other disc-like structure detectable in other tracers than those adopted here.

The results of Table 4 are visually represented in Fig. 29, where we plot the $L_{\mathrm{bol}} / M_{\mathrm{gas}}$ ratio as a function of distance. A few considerations are in order.

The most evolved source (i.e. the one with the highest value of $L_{\mathrm{bol}} / M_{\mathrm{gas}}$ ), G17.64, is also the only source without evidence for rotation. We note that the estimate of $L_{\mathrm{bol}} / M_{\mathrm{gas}}$ for G29.96
Table 4. Properties of the cores with velocity gradients detected with ALMA.

\begin{tabular}{lcccc}
\hline \hline Name & $\begin{array}{c}d \\
(\mathrm{kpc})\end{array}$ & $\begin{array}{c}H P B W^{a} \\
(\mathrm{AU})\end{array}$ & $\begin{array}{c}L_{\mathrm{bol}} / M_{\text {gas }} \\
\left(L_{\odot} / M_{\odot}\right)\end{array}$ & $\operatorname{disc}^{b}$ \\
\hline G17.64+0.16 & 2.2 & 332 & 300 & $\mathrm{~N}$ \\
G24.78+0.08 A1 & 7.7 & 1205 & 15 & $?$ \\
G29.96-0.02 HMC & 5.26 & 1168 & $300^{c}$ & $\mathrm{Y}$ \\
G31.41+0.31 & 7.9 & 1707 & 17 & $?$ \\
G345.49+1.47 M & 2.4 & 483 & 22 & $?$ \\
G345.50+0.35 M & 2.0 & 402 & 84 & $\mathrm{Y}$ \\
G345.50+0.35 S & 2.0 & 1217 & 84 & $\mathrm{Y}$ \\
AFGL 4176 & $d .2$ & 1205 & 120 & $Y$ \\
\hline
\end{tabular}

Notes. ${ }^{(a)}$ Half-power width of the synthesized beam at the distance of the source. ${ }^{(b)} \mathrm{Y}=$ disc candidate; ? = questionable disc; $\mathrm{N}=$ no evidence for a molecular disc. ${ }^{(c)}$ A significant fraction of $L_{\mathrm{bol}}$ from G29.96 is known to be contributed by the O5-O6 star associated with the nearby UC HII region (see Sect. 2). ${ }^{(d)}$ Revealed with ALMA by Johnston et al. (2015). The values of $L_{\text {bol }}\left(1.7 \times 10^{5} L_{\odot}\right)$ and $M_{\text {gas }}\left(1400 M_{\odot}\right)$ have been taken from Beltrán et al. (2007), after correcting for the different distance $(4.7 \mathrm{kpc}$ instead of $5.3 \mathrm{kpc})$ and dust temperature $(50 \mathrm{~K}$ instead of $30 \mathrm{~K})$.

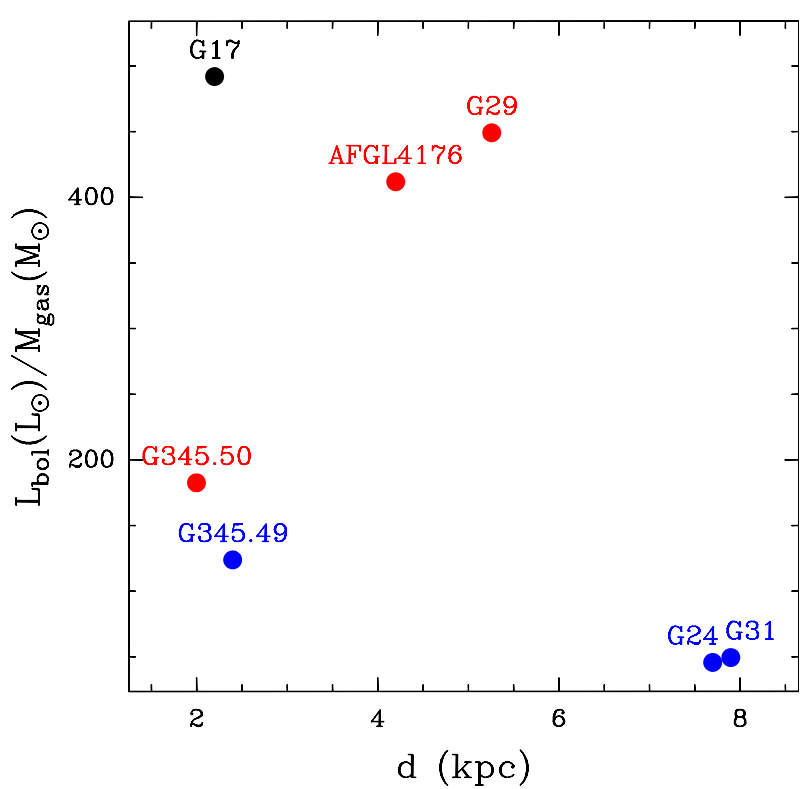

Fig. 29. Luminosity-to-mass ratio versus source distance for the six targets in our sample plus the disc around the $25 M_{\odot}$ star in AFGL 4176 detected by Johnston et al. (2015). Black, red, and blue points indicate, respectively, sources without evidence for discs, with disc candidates, and with questionable disc candidates (see Table 4).

is an upper limit due to the presence of the star ionizing the nearby UC HII region (see Sect. 2); therefore, the core is likely younger than suggested by the value of $L_{\mathrm{bol}} / M_{\mathrm{gas}}$. Although some hint of a disc seems present in the youngest sources (G31.41 and G24.78), the best disc candidates are G29.96, the two in G345.50, and, obviously, AFGL 4176, which have intermediate values of $L_{\mathrm{bol}} / M_{\mathrm{gas}}$.

The apparent lack of discs at a relatively later evolutionary stage is not surprising because our observations make use of high-density tracers, and the densest gas is gradually dispersed on a relatively large scale during the early evolution of a massive YSOs. Conversely, the marginal detection of discs in the youngest sources might be due to the massive envelopes 


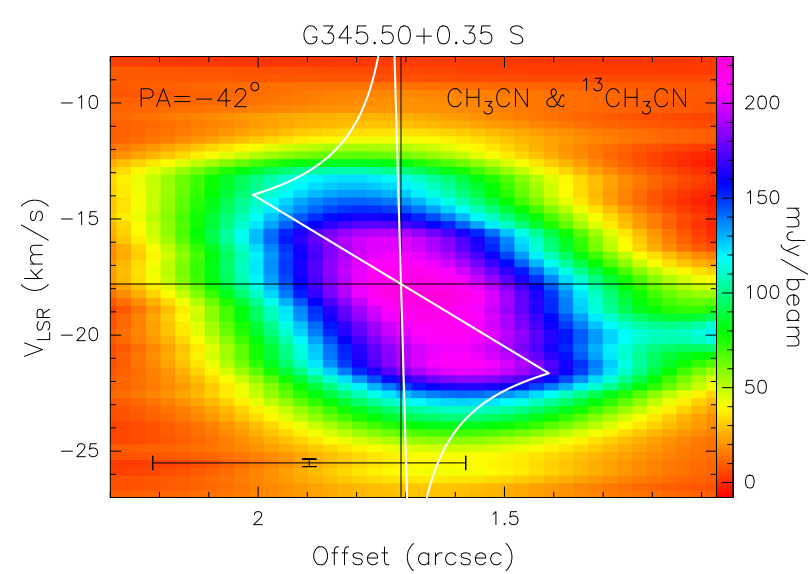

Fig. 30. Same as Fig. 23 for the $\mathrm{CH}_{3} \mathrm{CN}(12-11) K=2$ line in $\mathrm{G} 345.50$, after degrading the angular resolution by a factor 3.5 .

enshrouding the newly born stars; as discussed for G31.41 (see Sect. 5.4), their large opacity could confuse the picture and make it difficult to distinguish the embedded circumstellar discs. Also, stable Keplerian discs may start small and grow larger with time, as suggested for low-mass stars where only a few discs have been detected around Class 0 objects (Tobin et al. 2015; Murillo et al. 2013).

In this scenario, the best targets for our search are those YSOs where the molecular component of the disc is still important, but the surrounding envelope is less prominent. The influence of the environment is discussed in Sect. 5.6: the disc around G345.50 M appears much more perturbed (and hence more difficult to identify) than that around G345.50 S, because in the latter the circumstellar matter has a less complex distribution. In addition, as also discussed in Sect. 5.6, an age effect could be present because younger discs could be too small, making their detection challenging even for ALMA.

The previous considerations do not take into account another crucial parameter: the source distance. For obvious reasons one would expect the disc detection rate to decrease with increasing distance. If G345.50 were located at $7 \mathrm{kpc}$, the velocity gradient could still be detected, but the butterfly-shape of the PV plot in Fig. 23 would not be recognizable. This is demonstrated by Fig. 30, where the angular resolution of the original data has been degraded by a factor 3.5 (i.e. the ratio between $7 \mathrm{kpc}$ and the distance of G345.50, $2 \mathrm{kpc}$ ). Nevertheless, no obvious dependence of the detection rate with distance is seen in Table 4 and Fig. 29. In contrast, the only source (G17.64) without any evidence for a disc (from our ALMA data) is one of the closest, while one of the best candidates (G29.96) lies beyond $5 \mathrm{kpc}$.

With the caveat that the previous analysis is based on very low number statistics and a much larger number of cases has to be studied to reach statistical significance, we conclude that the detectability of a circumstellar disc around an O-type star appears sensitive to the evolutionary stage of the YSO. Under this hypothesis, the relatively low disc detection rate obtained in our observations is mostly due to the evolutionary phase of our targets: too late and too early are both unsuited - albeit for opposite reasons - and make it difficult to obtain a clear disc detection. This apparently negative argument can be converted into a positive one: if circumstellar discs are found at an intermediate evolutionary stage, they are also likely to exist in younger objects and thus be widespread in these luminous YSOs.

It remains to be understood if the stars at the centre of our disc candidates are indeed massive enough to be classified as
O-type stars and if the gas mass is consistent with Keplerian-like rotation.

Visual comparison between the PV plots and the white patterns ${ }^{7}$ corresponding to Keplerian rotation (see Figs. 16-23) suggests that a stellar mass of $\sim 10 M_{\odot}$ might suffice. This may be even less if stellar multiplicity is present, as expected for such luminous objects. However, a similar approach applied to the disc in AFGL 4176 studied by Johnston et al. (2015) suggests a stellar mass $\lesssim 10 M_{\odot}$, significantly less than that obtained from their model fit $\left(25 M_{\odot}\right)$. Moreover, if the disc is not edge-on the dynamical mass is a lower limit. For these reasons, we conclude that our findings are consistent with the detection of discs around stars in excess of $10 M_{\odot}$. To base this conclusion on a solid ground and, at the same time, obtain a precise estimate of other important parameters such as the disc mass, model fits analogous to that of Johnston et al. (2015) will be needed, a goal which is beyond the scope of the present study.

Finally, it is worth noting that to allow for Keplerian-like rotation, the disc mass should not exceed the stellar mass. It is thus important to verify that the masses of our cores are not much greater than any plausible stellar mass. An estimate of the gas mass can be obtained from the continuum emission if the dust temperature is known. The latter could be obtained by fitting the line emission of $\mathrm{HMC}$ tracers such as $\mathrm{CH}_{3} \mathrm{CN}$, under the assumption of equal gas and dust temperatures. A correct analysis should also take into account the existence of temperature gradients both in the putative disc and the surrounding envelope. While all this requires complex modelling, we can obtain a rough estimate of the mass using a plausible temperature from previous observations of some of our sources. For example, in G24.78 and G31.41 Beltrán et al. (2005) derive temperatures spanning from $60 \mathrm{~K}$ to $350 \mathrm{~K}$. For our sources, we assume a fiducial value of $200 \mathrm{~K}$, a dust absorption coefficient $\kappa_{\text {dust }}=1 \mathrm{~cm}^{2} \mathrm{~g}^{-1}$ at $1.4 \mathrm{~mm}$ (Ossenkopf \& Henning 1994), and a gas-to-dust mass ratio of 100 . We obtain gas masses ranging from $0.09 M_{\odot}$ to $330 M_{\odot}$, with only one source, G31.41, exceeding $19 M_{\odot}$. In conclusion, in all cases - apart from G31.41 - the gas mass is sufficiently small to warrant Keplerian rotation about a putative embedded O-type star. The exceptionally large size of the core in G31.41 (see Fig. 3) suggests that the estimated core mass of $330 M_{\odot}$ is probably dominated by the envelope, which is very difficult to separate from any embedded disc.

\section{Summary and conclusions}

We have reported on the results of ALMA observations of continuum and molecular line emission towards a selected sample of six high-mass star forming regions with luminosities typical of O-type stars. Our goal was to establish whether circumstellar discs around these stars are as common as in lower mass YSOs. After analysing the $\mathrm{CH}_{3} \mathrm{CN}$ and ${ }^{13} \mathrm{CH}_{3} \mathrm{CN}$ lines and (in three targets) the associated $\mathrm{SiO}$ line emission in the six regions, we find that in five of these at least one disc candidate is found and in two cases a clear bipolar outflow can be recognized.

The limited number of outflow detections suggests that in these massive objects collimated jets might be rare - perhaps short-lived - and the ejection phenomenon could be dominated by wide-angle winds, mostly resolved out in our high-resolution images. Whether this is an effect of age, intrinsic decollimation, precession, or presence of multiple stars cannot be decided on the basis of our data.

These patterns can be obtained, e.g., from Eq. (1) of Cesaroni et al. (2011), where only the rotation term must be considered. 
Further analysis of the velocity field by means of PV plots shows that in three objects a butterfly-shaped pattern is recognisable, which is believed to be a signpost of Keplerian-like ( $v_{\text {rot }} \propto R^{-\alpha}$, with $\alpha>0$ ) rotation, whereas in the other three cores only marginal evidence for this pattern is seen.

With the caveat that a larger number of objects has to be studied to base any final statement on a solid statistical ground, we conclude that unveiling the presence of circumstellar discs around O-type stars may be extremely challenging even for ALMA because young, deeply embedded discs may be masked by the surrounding optically thick envelope, while evolved ones could have lost most of their molecular component. Only discs around YSOs in an intermediate evolutionary phase could be detectable. In this respect, the study of more complex molecules, with lower abundances than $\mathrm{CH}_{3} \mathrm{CN}$ and its isotopologues, could help in tracing the disc thanks to their presumably lower line opacities.

The lesson to learn from our findings is that too young and too old are both unsuited, and future searches for discs around young O-type YSOs should take this caveat into consideration.

Acknowledgements. Á.S.M. and P.S. thank the Deutsche Forschungsgemeinschaft (DFG) for funding support via the collaborative research grant SFB 956, project A6. T.P. acknowledges the Deutsche Forschungsgemeinschaft (DFG) for funding through the SPP 1573 "The Physics of the Interstel lar Medium". R.G.-M. acknowledges support from UNAM-DGAPA-PAPIIT IA101715. R.K. acknowledges financial support via the Emmy Noether Research Group on "Accretion Flows and Feedback in Realistic Models of Massive Star Formation" funded by the German Research Foundation (DFG) under grant no. KU 2849/3-1. This paper makes use of the following ALMA data: ADS/JAO.ALMA\#2013.1.00489.S. ALMA is a partnership of ESO (representing its member states), NSF (USA), and NINS (Japan), together with NRC (Canada), and NSC and ASIAA (Taiwan), in cooperation with the Republic of Chile. The Joint ALMA Observatory is operated by ESO, AUI/NRAO, and NAOJ. This work also used observations made with the Spitzer Space Telescope, which is operated by the Jet Propulsion Laboratory, California Institute of Technology, under a contract with NASA. M.S.N.K. is supported by a H2020 MarieCurie IEF (GESTATE 661249) funded by the European Research Comission.

\section{References}

Bachiller, R., \& Pérez Gutiérrez, M. 1997, ApJ, 487, L93

Beltrán, M. T., Cesaroni, R., Neri, R., et al. 2004, ApJ, 601, L187

Beltrán, M. T., \& de Wit, W. J. 2016, A\&ARv, 24, 6

Beltrán, M. T., Cesaroni, R., Neri, R., et al. 2005, A\&A, 435, 901

Beltrán, M. T., Brand, J., Cesaroni, R., et al. 2006, A\&A, 447, 221

Beltrán, M. T., Cesaroni, R., Moscadelli, L., \& Codella, C. 2007, A\&A, 471, L13

Beltrán, M. T., Cesaroni, R., Neri, R., \& Codella, C. 2011a, A\&A, 525, A151

Beltrán, M. T., Cesaroni, R., Zhang, Q., et al. 2011b, A\&A, 532, A91

Beltrán, M. T., Olmi, L., Cesaroni, R., et al. 2013, A\&A, 552, A123

Beltrán, M. T., Sánchez-Monge, Á., Cesaroni, R., et al. 2014, A\&A, 571, A52

Beuther, H., Zhang, Q., Bergin, E. A., et al. 2007, A\&A, 468, 1045

Bonnell, I. A., \& Bate, M. R. 2006, MNRAS, 370, 488

Cesaroni, R., Churchwell, E., Hofner, P., Walmsley, C. M., \& Kurtz, S. 1994a, A\&A, 288, 903

Cesaroni, R., Olmi, L., Walmsley, C. M., Churchwell, E., \& Hofner, P. 1994b, ApJ, 435, L137

Cesaroni, R., Neri, R., Olmi, L., et al. 2005, A\&A, 434, 1039

Cesaroni, R., Galli, D., Lodato, G., Walmsley, C. M., \& Zhang, Q. 2007, in Protostars and Planets V, eds. B. Reipurth, D. Jewitt, \& K. Keil (Tucson: Univ. of Arizona Press), 197

Cesaroni, R., Hofner, P., Araya, E., \& Kurtz, S. 2010, A\&A, 509, 50

Cesaroni, R., Beltrán, M. T., Zhang, Q., Beuther, H., \& Fallscheer, C. 2011 A\&A, 533, A73

Cesaroni, R., Galli, D., Neri, R., \& Walmsley, C. M. 2014, A\&A, 566, A73

Cesaroni, R., Pestalozzi, M., Beltrán, M. T., et al. 2015, A\&A, 579, A71
Codella, C., Beltrán, M. T., Cesaroni, R., et al. 2013, A\&A, 550, A81 Condon, J. J., Cotton, W. D., Greisen, E. W., et al. 1998, AJ, 115, 1693 Contreras, Y., Schuller, F., Urquhart, J. S., et al. 2013, A\&A, 549, A45 Churchwell, E., Babler, B. L., Meade, M. R., et al. 2009, PASP, 121, 213 De Buizer, J. M., Watson, A. M., Radomski, J. T., Piña, R. K., \& Telesco, C. M. 2002, ApJ, 564, L101

Elia, D., Schisano, E., Molinari, S., et al. 2010, A\&A, 518, L97

Faúndez, S., Bronfman, L., Garay, G., et al. 2004, A\&A, 426, 97

Forster, J. R., \& Caswell, J. L. 1989, A\&A, 213, 339

Garay, G., Brooks, K. J., Mardones, D., \& Norris, R. P. 2006, ApJ, 651, 914

Gueth, F., Guilloteau, S., \& Bachiller, R. 1998, A\&A, 333, 287

Ginsburg, A., Glenn, J., Rosolowsky, E., et al. 2013, ApJS, 208, 14

Guzmán, A. E., Garay, G., Brooks, K. J. 2010, ApJ, 725, 734

Guzmán, A. E., Garay, G., Brooks, K. J., Rathborne, J., \& Güsten, R. 2011, ApJ, 736,150

Guzmán, A. E., Garay, G., Rodríguez, L. F., et al. 2016, ApJ, 826, 208

Johnston, K. G., Robitaille, T. P., Beuther, H., et al. 2015, ApJ, 813, L19

Kastner, J. H., Weintraub, D. A., Snell, R. L., et al. 1994, ApJ, 425, 695

Keto, E. 2002, ApJ, 580, 980

Keto, E. 2003, ApJ, 599, 1196

Keto, E. 2007, ApJ, 666, 976

Klassen, M., Pudritz, R. E., Kuiper, R., Peters, T., \& Banerjee, R. 2016, ApJ, 823,28

Krumholz, M. R., Klein, R. I., \& McKee, C. F. 2007a, ApJ, 656, 959

Krumholz, M. R., Klein, R. I., \& McKee, C. F. 2007b, ApJ, 665, 478

Krumholz, M. R., Klein, R. I., McKee, C. F., Offner, S. S. R., \& Cunningham, A. J. 2009, Science, 323, 754

Kuiper, R., Klahr, H., Beuther, H., \& Henning, Th. 2010, ApJ, 722, 1556

Kuiper, R., Klahr, H., Beuther, H., \& Henning, Th. 2011, ApJ, 732, 20

Kuiper, R., Turner, N. J., \& Yorke, H. W. 2016, ApJ, 832, 40

Lumsden, S. L., Hoare, M. G., Urquhart, J. S., et al. 2013, ApJS, 208, 11

Martín-Hernández, N. L., Bik, A., Kaper, L., Tielens, A. G. G. M., \& Hanson, M. M. 2003, A\&A, 405, 175

Maud, L. T., Lumsden, S. L., Moore, T. J. T., et al. 2015a, MNRAS, 452, 637

Maud, L. T., Moore, T. J. T., Lumsden, S. L., et al. 2015b, MNRAS, 453, 645

McKee, C. F., \& Tan, J. C. 2003, ApJ, 585, 850

Mayen-Gijon, J. M., Anglada, G., Osorio, M., et al. 2014, MNRAS, 437, 3766

Menten, K. M., \& van der Tak, F. F. S. 2004, A\&A, 414, 289

Moisés, A. P., Damineli, A., Figuerêdo, E., et al. 2011, MNRAS, 411, 705

Molinari, S., Swinyard, B., Bally, J., et al. 2010, PASP, 122, 314

Molinari, S., Schisano, E., Elia, D., et al. 2016, A\&A, 591, A149

Moscadelli, L., Li, J. J., Cesaroni, R., Sanna, A., Xu, Y., \& Zhang, Q. 2013, A\&A, 549, A122

Mottram, J. C., Hoare, M. G., Urquhart, J. S., et al. 2011a, A\&A, 525, A149

Mottram, J. C., Hoare, M. G., Davies, B., et al. 2011b, ApJ, 730, L33

Murillo, N. M., Lai, S.-P., Bruderer, S., Harsono, D., van Dishoeck, E. F. 2013, A\&A, 560, 103

Ossenkopf, V., \& Henning, Th. 1994, A\&A, 291, 943

Peters, T., Banerjee, R., Klessen, R. S., et al. 2010, ApJ, 711, 1017

Peters, T., Banerjee, R., Klessen, R. S., \& Mac Low, M.-M. 2011, ApJ, 729, 72

Peters, T., Klaassen, P. D., Mac Low, M.-M., et al. 2014, ApJ, 788, 14

Rosen, A. L., Krumholz, M. R., \& Ramirez-Ruiz, E. 2012, ApJ, 748, 97

Sánchez-Monge, Á., Cesaroni, R., Beltrán, M. T., et al. 2013, A\&A, 552, L10

Sánchez-Monge, Á., Beltrán, M. T., Cesaroni, R., et al. 2014, A\&A, 569, A11

Sánchez-Monge, Á., Schilke, P., Ginzburg, A., Cesaroni, R., \& Schmiedeke, A. 2017, A\&A, submitted

Schuller, F., Menten, K. M., Contreras, Y., et al. 2009, A\&A, 504, 415

Sollins, P. K., Hunter, T. R., Battat, J., et al. 2004, ApJ, 616, L35

Tan, J. C., Beltrán, M. T., Caselli, P., et al. 2014, in Protostars and Planets VI, eds. H. Beuther, R. S. Klessen, C. P. Dullemond, \& T. Henning (Tucson: Univ. of Arizona Press), 149

Tobin, J. J., Looney, L. W., Wilner, D. J., et al. 2015, ApJ, 805, 125

Urquhart, J. S., Busfield, A. L. Hoare, M. G., et al. 2008, A\&A, 487, 253

Urquhart, J. S., Hoare, M. G., Purcell, C. R., et al. 2009, A\&A, 501, 539

Vacca, W. D., Garmany, C. D., \& Shull, M. 1996, ApJ, 460, 914

van der Tak, F. F. S., van Dishoeck, E. F., Evans, N. J., II, Blake, G. A. 2000, ApJ, 537, 283

Vig, S., Cesaroni, R., Testi, L., Beltrán, M. T., \& Codella, C. 2008, A\&A, 488, 605

Zapata, L. A., Palau, A., Galván-Madrid, R., et al. 2015, MNRAS, 447, 1826

Zhang, Y., Tan, J. C., De Buizer, J. M., et al. 2013, ApJ, 767, 58

Zhang, B., Moscadelli, L., Sato, M., Reid, M. J., et al. 2014, ApJ, 781, 89 
1 INAF, Osservatorio Astrofisico di Arcetri, Largo E. Fermi 5, 50125 Firenze, Italy e-mail: cesa@arcetri.astro.it

2 I. Physikalisches Institut, Universität zu Köln, Zülpicher Strasse 77, 50937 Köln, Germany

3 School of Physics and Astronomy, University of Leeds, West Yorkshire, Leeds LS2 9JT, UK

${ }^{4}$ ALLEGRO/Leiden Observatory, Leiden University, PO Box 9513, 2300 RA Leiden, the Netherlands

5 Max Planck Institute for Astronomy, Königstuhl 17, 69117 Heidelberg, Germany

${ }^{6}$ Kapteyn Astronomical Institute, University of Groningen, 9700 AV, Groningen, The Netherlands

7 Max-Planck-Institut für Radioastronomie, Auf dem Hügel 69, 53121 Bonn, Germany

8 Hamburger Sternwarte, Universität Hamburg, 21029 Hamburg, Germany

9 Jodrell Bank Centre for Astrophysics, School of Physics and Astronomy, The University of Manchester, Oxford Road, Manchester M13 9PL, UK

10 UK ALMA Regional Centre Node, The University of Manchester, Manchester M13 9PL, UK

11 Instituto de Radioastronomía y Astrofísica, UNAM, Apdo. Postal 3-72 (Xangari), 58089 Morelia, Michoacán, México
12 Department of Astrophysics/IMAPP, Radboud University, PO Box 9010, 6500 GL Nijmegen, The Netherlands

13 School of Physics and Astronomy, University of Leeds, Leeds LS2 9JT, UK

14 UK Astronomy Technology Centre, Royal Observatory Edinburgh, Blackford Hill, Edinburgh EH9 3HJ, UK

15 Institut für Astronomie und Astrophysik, Universität Tübingen, Auf der Morgenstelle 10, 72076 Tübingen, Germany

16 Centre for Astrophysics, University of Hertfordshire, College Lane, Hatfield, AL10 9AB, UK

17 Instituto de Astrofísica e Ciências do Espaço, Universidade do Porto, CAUP, Rua das Estrelas, 4150-762 Porto, Portugal

18 School of Physics and Astronomy, University of Leeds, West Yorkshire, Leeds LS2 9JT, UK

19 Max-Planck-Institut für Astrophysik, Karl-Schwarzschild-Str. 1, 85741 Garching, Germany

${ }^{20}$ European Southern Observatory, Karl-Schwarzschild-Str. 2, 85748 Garching bei München, Germany

21 SRON Netherlands Institute for Space Research, Landleven 12, 9747 AD, Groningen, The Netherlands

22 Indian Institute of Space science and Technology, 695547 Thiruvananthapuram, India

${ }^{23}$ Dublin Institute of Advanced Studies, Fitzwilliam Place 31, Dublin 2, Ireland

24 SOFIA Science Center, Deutsches SOFIA Institut, NASA Ames Research Center, Moffett Field, CA, 94035, USA 